\title{
An Impulsively Controlled Three-Species Prey-Predator Model with Stage Structure and Birth Pulse for Predator
}

\author{
Yanyan Hu, ${ }^{1,2}$ Mei Yan, ${ }^{2}$ and Zhongyi Xiang ${ }^{1,2}$ \\ ${ }^{1}$ Key Laboratory of Biologic Resources Protection and Utilization of Hubei Province, Enshi, Hubei 445000, China \\ ${ }^{2}$ Department of Mathematics, Hubei University for Nationalities, Enshi, Hubei 445000, China \\ Correspondence should be addressed to Zhongyi Xiang; yfxiang2007@163.com
}

Received 22 April 2015; Accepted 21 June 2015

Academic Editor: Alicia Cordero

Copyright (c) 2015 Yanyan Hu et al. This is an open access article distributed under the Creative Commons Attribution License, which permits unrestricted use, distribution, and reproduction in any medium, provided the original work is properly cited.

\begin{abstract}
We investigate the dynamic behaviors of a two-prey one-predator system with stage structure and birth pulse for predator. By using the Floquet theory of linear periodic impulsive equation and small amplitude perturbation method, we show that there exists a globally asymptotically stable two-prey eradication periodic solution when the impulsive period is less than some critical value. Further, we study the permanence of the investigated model. Our results provide valuable strategy for biological economics management. Numerical analysis is also inserted to illustrate the results.
\end{abstract}

\section{Introduction}

In the natural world, the predator-prey relationship is one of the important interactions among species, and it has been extensively studied by many authors [1-7] because of its universal existence. The following two-prey, one-predator model was studied by many works [8-10]:

$$
\begin{aligned}
& \dot{x}_{1}=x_{1}\left(b_{1}-x_{1}-\alpha x_{2}-r_{1} y\right), \\
& \dot{x}_{2}=x_{2}\left(b_{2}-\beta x_{1}-x_{2}-r_{2} y\right), \\
& \dot{y}=y_{1}\left(-b_{3}+d r_{1} x_{1}+d r_{2} x_{2}\right),
\end{aligned}
$$

where $x_{i}(t)(i=1,2)$ and $y(t)$ are densities of the prey population and the predator population, respectively. $r_{i}>$ $0(i=1,2)$ are coefficients of decrease of prey due to predation; $b_{i}>0(i=1,2,3)$ are intrinsic rates of increase or decrease. $\alpha>0, \beta>0$ are parameters representing competitive effects between two prey species; $d>0$ is an equal transformation rate of the predator.

As we know, life history often occurs in natural ecological environments which has significant morphological and behavioral differences between immature and mature species; the dynamics of stage-structured prey-predator system has been widely studied [11-13]. Recently, many impulsive differential equation models or hybrid dynamical systems have been proposed to model the introduction of a periodic IPM strategy [14, 15]; Xiang and Song [16] proposed impulsive prey-dependent consumption two-prey one-predator models with stage structure for the predator, which combined the biological control and chemical control:

$$
\begin{aligned}
\dot{x}_{1}(t)= & b_{1} x_{1}(t)\left(1-x_{1}(t)\right)-\alpha x_{1}(t) x_{2}(t) \\
& -r_{1} x_{1}(t) y_{2}(t), \\
\dot{x}_{2}(t)= & b_{2} x_{2}(t)\left(1-x_{2}(t)\right)-\beta x_{1}(t) x_{2}(t) \\
& -r_{2} x_{2}(t) y_{2}(t), \\
\dot{y}_{1}(t)= & \frac{\lambda_{1} r_{1} x_{1}(t) y_{2}(t)}{1+h_{1} r_{1} x_{1}(t)}+\frac{\lambda_{2} r_{2} x_{2}(t) y_{2}(t)}{1+h_{2} r_{2} x_{2}(t)} \\
& -(m+\mu) y_{1}(t), \\
\dot{y}_{2}(t)= & m y_{1}(t)-\mu y_{2}(t),
\end{aligned}
$$$$
t \neq n T \text {, }
$$

$\Delta x_{1}(t)=-E_{1} x_{1}(t)$, 


$$
\begin{aligned}
& \Delta x_{2}(t)=-E_{2} x_{2}(t), \\
& \Delta y_{1}(t)=p_{1}, \\
& \Delta y_{2}(t)=p_{2},
\end{aligned}
$$$$
t=n T \text {. }
$$

The biological meanings of the parameters in (2) can be seen in [16]. As far as the population dynamics is concerned, most models often considered that the population reproduces throughout all year. However, many species give birth seasonally or in regular pules. Particular examples of birth pulse type are $\Delta N=B(N) N$. The model discussed in [17] considered the birth pulse $\Delta N=B(N) N, B(N)=b-C N^{\theta}$. A birth pulse type $\Delta N=B(N) N$ with $B(N)=b e^{N}$ and $B(N)=p /\left(q+N^{n}\right)$ are assumed in [18]. Comparatively, we consider birth pulse $\Delta N=N(a-b N)$.

In view of birth pulse and impulsive control strategy, we formulate the following two prey-predator models with stagestructure and birth pulse for predator:

$$
\begin{aligned}
\dot{x}_{1}(t)= & x_{1}(t)\left(b_{1}-x_{1}(t)\right)-\alpha x_{1}(t) x_{2}(t) \\
& -r_{1} x_{1}(t) y_{1}(t)-r_{2} x_{1}(t) y_{2}(t), \\
\dot{x}_{2}(t)= & x_{2}(t)\left(b_{2}-x_{2}(t)\right)-\beta x_{1}(t) x_{2}(t) \\
& -r_{3} x_{2}(t) y_{1}(t)-r_{4} x_{2}(t) y_{2}(t), \\
\dot{y}_{1}(t)= & \lambda_{1} r_{1} x_{1}(t) y_{1}(t)+\lambda_{3} r_{3} x_{2}(t) y_{1}(t) \\
& -(m+\mu) y_{1}(t), \\
\dot{y}_{2}(t)= & \lambda_{2} r_{2} x_{1}(t) y_{2}(t)+\lambda_{4} r_{4} x_{2}(t) y_{2}(t)+m y_{1}(t) \\
& -\mu y_{2}(t), \\
\Delta x_{1}(t)= & -p_{1} x_{1}(t), \\
\Delta x_{2}(t)= & -p_{2} x_{2}(t), \\
\Delta y_{1}(t)= & -p_{3} y_{1}(t), \\
\Delta y_{2}(t)= & -p_{4} y_{2}(t),
\end{aligned}
$$

$$
t=(n+l) T, n=1,2, \ldots
$$

$\Delta x_{1}(t)=0$,

$\Delta x_{2}(t)=0$,

$\Delta y_{1}(t)=y_{2}(t)\left(a-b y_{2}(t)\right)$

$\Delta y_{2}(t)=0$, where $x_{i}(t)(i=1,2)$ are densities of the prey population and $y_{1}(t)$ and $y_{2}(t)$ represent the densities of the immature and the mature predator population, respectively. $r_{i}>0(i=$ $1,2,3,4)$ are coefficients of decrease of prey due to predation, $\alpha>0, \beta>0$ are parameters representing competitive effects between two prey species, and $\lambda_{i}>0(i=1,2,3,4)$ represent the rate of conversion of nutrients into the predators. The maturity is $m$, which determines the mean length of the juvenile period; $\mu$ is the natural death rate of the predator population. $\Delta y_{1}(t)=y_{2}(t)\left(a-b y_{2}(t)\right)$ is birth pulse at $t=$ $(n+1) T$ as intrinsic rate of natural increase and density dependence rate of predator population are denoted by $a$, $b$, respectively. $a / b$ is the carrying capacity of the predator population. $0 \leq l \leq 1,0 \leq p_{i} \leq 1(i=1,2,3,4)$ represent the fraction of prey and predators which die due to the pesticide at $t=(n+l) T, n \in Z_{+}$.

The organization of this paper is as follows. In the next section, some important lemmas are presented. Sections 3 and 4 give the global asymptotical stability of the prey eradication periodic solution and permanence for system (3). Numerical analysis is displayed in Section 5. Finally, a brief discussion is given to conclude this work.

\section{Preliminaries}

In this section, we will give some definitions, notations, and some lemmas which will be useful for our main results.

Let $R_{+}=[0, \infty), R_{+}^{4}=\left\{x \in R^{4}: x>0\right\}$. Thus $\Omega=$ int $R_{+}^{4}$; let $Z_{+}$be the set of all nonnegative integers. Denote $f=\left(f_{1}, f_{2}, f_{3}, f_{4}\right)$, the map defined by the right hand side of the first four equations of system (3). Let $V: R_{+} \times R_{+}^{4} \mapsto R_{+}$; then $V$ is said to belong to class $V \in V_{0}$ if

(i) $V$ is continuous in $(n T,(n+l) T] \times R_{+}^{4}$ and $((n+$ l)T, $(n+1) T] \times R_{+}^{4}$ for each, $x \in R_{+}^{4}, n \in Z_{+}$, $\lim _{(t, y) \rightarrow\left((n+l) T^{+}, x\right)} V(t, y)=V\left((n+l) T^{+}, x\right)$ and $\lim _{(t, y) \rightarrow\left((n+1) T^{+}, x\right)} V(t, y)=V\left((n+1) T^{+}, x\right)$ existing;

(ii) $V$ is locally Lipschitzian in $x$.

Definition 1. Consider $V \in V_{0}$; then, for $(t, x) \in(n T,(n+$ l) $T] \times R_{+}^{4}$ and $(t, x) \in((n+l) T,(n+1) T] \times R_{+}^{4}$, the upper right derivative of $V(t, x)$ with respect to the impulsive differential system (3) is defined as $D^{+} V(t, x)=\lim _{h \rightarrow 0^{+}} \sup (1 / h)[V(t+$ $h, x+h f(t, x))-V(t, x)]$.

The solution of system (3), denoted by $x(t)=$ $\left(x_{1}(t), x_{2}(t), y_{1}(t), y_{2}(t)\right): R_{+} \mapsto R_{+}^{4}$, is continuously differentiable on $(n T,(n+l) T]$ and $((n+l) T,(n+1) T]$, $n \in Z_{+}, 0 \leq l \leq 1$.

Obviously the smoothness properties of $f$ guarantee the global existence and uniqueness of solutions of system (3); for details see [19]. The following lemma is obvious.

Lemma 2. Suppose $x(t)$ is a solution of (3) with $x\left(0^{+}\right) \geq 0$; then $x(t) \geq 0$ for all $t \geq 0$. And further $x(t)>0, t \geq 0$, if $x\left(0^{+}\right)>0$. 
Make a notation as

$$
p_{4}^{*}=\frac{\left[e^{-m T}\left(1-p_{3}\right)+p_{3}\left(e^{-m T}-e^{-m l T}\right) a\right]-e^{\mu T}+1+a\left(1-e^{-m T}\right)-\left(1-p_{3}\right) e^{-(m+\mu) T}}{1+a\left(1-e^{-m T}\right)-\left(1-p_{3}\right) e^{-(m+\mu) T}} .
$$

If $x_{1}(t)=0, x_{2}(t)=0$, then system (3) can be rewriten to

$$
\begin{aligned}
& \dot{y}_{1}(t)=-(m+\mu) y_{1}, \\
& \dot{y}_{2}(t)=m y_{1}(t)-\mu y_{2}(t),
\end{aligned}
$$$$
t \neq(n+l) T, \quad t \neq(n+1) T
$$

$\Delta y_{1}(t)=-p_{3} y_{1}(t)$

$$
\Delta y_{2}(t)=-p_{4} y_{2}(t)
$$

$$
t=(n+l) T
$$$$
\Delta y_{1}(t)=y_{2}(t)\left(a-b y_{2}(t)\right),
$$$$
\Delta y_{2}(t)=0 \text {, }
$$

$$
t=(n+1) T \text {. }
$$

It is easy to obtain the analytic solution of system (5) between pulses:

$$
\begin{aligned}
& y_{1}(t)= \begin{cases}y_{1}\left(n T^{+}\right) e^{-(m+\mu)(t-n T)}, & t \in(n T,(n+l) T], \\
y_{1}\left((n+l) T^{+}\right) e^{-(m+\mu)(t-(n+l) T)}, & t \in((n+l) T,(n+1) T],\end{cases} \\
& y_{2}(t)= \begin{cases}e^{-\mu(t-n T)}\left[y_{2}\left(n T^{+}\right)+y_{1}\left(n T^{+}\right)\left(1-e^{-m(t-n T)}\right)\right], & t \in(n T,(n+l) T], \\
e^{-\mu(t-(n+l) T)}\left[y_{2}\left((n+l) T^{+}\right)+y_{1}\left((n+l) T^{+}\right)\left(1-e^{-m(t-(n+l) T)}\right)\right], & t \in((n+l) T,(n+1) T] .\end{cases}
\end{aligned}
$$

Considering (5), we have the stroboscopic map of system

$$
\begin{aligned}
& y_{1}\left((n+1) T^{+}\right)=a e^{-\mu T}\left(1-p_{4}\right) y_{2}\left(n T^{+}\right) \\
& +\left\{a \left[\left(1-p_{4}\right) e^{-\mu T}\left(1-e^{-m l T}\right)\right.\right. \\
& \left.+\left(1-p_{3}\right) e^{-(\mu+m l) T}\left(1-e^{(1-l) m T}\right)\right]+\left(1-p_{3}\right) \\
& \left.\quad \cdot e^{-(m+\mu) T}\right\} y_{1}\left(n T^{+}\right)-b\left\{e^{-\mu T}\left(1-p_{4}\right) y_{2}\left(n T^{+}\right)\right. \\
& +\left[\left(1-p_{4}\right) e^{-\mu T}\left(1-e^{-m l T}\right)\right. \\
& \left.\left.+\left(1-p_{3}\right) e^{-(\mu+m l) T}\left(1-e^{(1-l) m T}\right)\right] y_{1}\left(n T^{+}\right)\right\}, \\
& y_{2}\left((n+1) T^{+}\right)=e^{-\mu T}\left(1-p_{4}\right) y_{2}\left(n T^{+}\right)+\left[\left(1-p_{4}\right)\right. \\
& \quad \cdot e^{-\mu T}\left(1-e^{-m l T}\right)+\left(1-p_{3}\right) e^{-(\mu+m l) T}(1 \\
& \left.\left.\quad-e^{(1-l) m T}\right)\right] y_{1}\left(n T^{+}\right) .
\end{aligned}
$$

For convenience, we choose $A=e^{-\mu T}\left(1-p_{4}\right)>0, B=$ $\left(1-p_{3}\right) e^{-(\mu+m l) T}>0$, and $C=\left(1-p_{4}\right) e^{-\mu T}\left(1-e^{-m l T}\right)+(1-$ $\left.p_{3}\right) e^{-(\mu+m l) T}\left(1-e^{(1-l) m T}\right)>0$; the following two equivalence relations are calculated to be

$$
\begin{aligned}
& p_{4}>p_{4}^{*} \Longleftrightarrow B+a C-1-A B+A<0, \\
& p_{4}<p_{4}^{*} \Longleftrightarrow B+a C-1-A B+A>0 .
\end{aligned}
$$

The two fixed points of (5) are obtained as $G_{0}(0,0)$ and $G\left(y_{1}^{*}, y_{2}^{*}\right)$, where

$$
\begin{aligned}
& y_{1}^{*}=\frac{(B+a C-1-A B+A)(1-A)}{b C^{2}}, \quad p_{4}<p_{4}^{*}, \\
& y_{2}^{*}=\frac{(B+a C-1-A B+A)}{b C}, \quad p_{4}<p_{4}^{*} .
\end{aligned}
$$

Lemma 3. (i) If $p_{4}>p_{4}^{*}$, the fixed point $G_{0}(0,0)$ is globally asymptotically stable.

(ii) If $p_{4}<p_{4}^{*}$, the fixed point $G\left(y_{1}^{*}, y_{2}^{*}\right)$ is globally asymptotically stable.

Proof. For convenience, we make a notation as $\left(y_{1}^{n}, y_{2}^{n}\right)=$ $\left(y_{1}^{*}, y_{2}^{*}\right)$. Linear form (5) can be written as

$$
\left(\begin{array}{c}
y_{1}^{n+1} \\
y_{2}^{n+1}
\end{array}\right)=J\left(\begin{array}{c}
y_{1}^{n} \\
y_{2}^{n}
\end{array}\right)
$$

Obviously, the near dynamics of $G_{0}(0,0)$ and $G\left(y_{1}^{*}, y_{2}^{*}\right)$ are determined by linear system (10). The stabiles of $G_{0}(0,0)$ and $G\left(y_{1}^{*}, y_{2}^{*}\right)$ are determined by the eigenvalue of $J$ less than 1. If $J$ satisfies the Jury criteria [20], we can know that the eigenvalue of $J$ is less than 1 :

$$
1-\operatorname{tr} J+\operatorname{det} J>0
$$


where

$$
=\left(\begin{array}{cc}
B+a C-2 b C^{2} y_{1}^{*}-2 b A C y_{2}^{*} & a A-2 b A^{2} y_{2}^{*}-2 b A C y_{1}^{*} \\
C & A
\end{array}\right) .
$$

(i) If $p_{4}>p_{4}^{*}$, then $B+a C-1-A B+A<0, G_{0}(0,0)$ is the unique fixed point of system (5); we have

$$
J_{1}=\left.J\right|_{(0,0)}\left(\begin{array}{cc}
B+a C & a A \\
C & A
\end{array}\right) .
$$

Calculating $1-\operatorname{tr} J_{1}+\operatorname{det} J_{1}=1-A-B+A B-a C>0$, and from the Jury criteria $G_{0}(0,0)$ is locally stable, and then it is globally asymptotically stable. (ii) If $p_{4}<p_{4}^{*}$, then $B+a C-1-A B+A>0, G_{0}(0,0)$ is unstable. For $B+a C-1-A B+A>0, G\left(y_{1}^{*}, y_{2}^{*}\right)$ exists, we have

$$
\begin{aligned}
J_{2} & =\left.J\right|_{\left(y_{1}^{*}, y_{2}^{*}\right)} \\
& \cdot\left(\begin{array}{cc}
B+a C-2 b C\left(C y_{1}^{*}+A y_{2}^{*}\right) & a A-2 b A\left(C y_{1}^{*}+A y_{2}^{*}\right) \\
C & A
\end{array}\right) .
\end{aligned}
$$

Consider $1-\operatorname{tr} J+\operatorname{det} J=1-A-B+A B-a C>0$. From the Jury criteria $G\left(y_{1}^{*}, y_{2}^{*}\right)$ is locally stable, and then it is globally asymptotically stable. The proof is completed.

Summarizing the above results, we have the following theorems.

Theorem 4. The triviality periodic solution $G_{0}(0,0)$ is stable if $p_{4}>p_{4}^{*}$; otherwise, if $p_{4}<p_{4}^{*}$, the triviality periodic solution $G_{0}(0,0)$ is unstable, and the periodic solution $G\left(y_{1}^{*}, y_{2}^{*}\right)$ is globally asymptotically stable.

Correspondingly, system (5) has a globally asymptotically stable positive periodic solution $\left(\widetilde{y}_{1}(t), \widetilde{y}_{2}(t)\right)$, where

$$
\begin{aligned}
& \tilde{y}_{1}(t)= \begin{cases}y_{1}^{*} e^{-(m+\mu)(t-n T)}, & t \in(n T,(n+l) T], \\
y_{1}^{*} e^{-(m+\mu) l T} e^{-(m+\mu)(t-(n+l) T)}, & t \in((n+l) T,(n+1) T],\end{cases} \\
& \tilde{y}_{2}(t)= \begin{cases}e^{-\mu(t-n T)}\left[y_{2}^{*}+y_{1}^{*}\left(1-e^{-m(t-n T)}\right)\right], & t \in(n T,(n+l) T], \\
e^{-\mu(t-(n+l) T)}\left[e^{-\mu l T}\left[y_{2}^{*}+y_{1}^{*}\left(1-e^{-m l T}\right)\right]+y_{1}^{*} e^{-(m+\mu) l T}\left(1-e^{-m(t-(n+l) T)}\right)\right], & t \in((n+l) T,(n+1) T] .\end{cases}
\end{aligned}
$$

Lemma 5. There exists a constant $M>0$ such that $x_{i}(t) \leq$ $M, y_{i}(t) \leq M(i=1,2)$, for each positive solution $x(t)=$ $\left(x_{1}(t), x_{2}(t), y_{1}(t), y_{2}(t)\right)$ of $(5)$ with all t large enough.

Proof. Define a function $V(t, x)$ such that $V(t, x)=$ $\sum_{i=1}^{2}\left(\lambda x_{i}(t)+y_{i}(t)\right)$, where $\lambda=\max \left\{\lambda_{1}, \lambda_{2}, \lambda_{3}, \lambda_{4}\right\}$. When $t \neq(n+l) T, t \neq(n+1) T$, we have

$$
\begin{aligned}
D^{+} V(t, x)+\mu V(t, x) \\
\leq-b_{1}\left(x_{1}-\frac{b_{1}+\mu}{2 b_{1}}\right)^{2}+\frac{\left(b_{1}+\mu\right)^{2}}{4 b_{1}} \\
\quad-b_{2}\left(x_{1}-\frac{b_{2}+\mu}{2 b_{2}}\right)^{2}+\frac{\left(b_{2}+\mu\right)^{2}}{4 b_{2}} \\
\leq \frac{\left(b_{1}+\mu\right)^{2}}{4 b_{1}}+\frac{\left(b_{2}+\mu\right)^{2}}{4 b_{2}} \triangleq M_{1} .
\end{aligned}
$$

When $t=(n+l) T$,

$$
\begin{aligned}
V\left((n+l) T^{+}\right)= & \lambda x_{1}\left((n+l) T^{+}\right)+\lambda x_{2}\left((n+l) T^{+}\right) \\
& +y_{1}\left((n+l) T^{+}\right)+y_{2}\left((n+l) T^{+}\right) \\
\leq & V((n+l) T),
\end{aligned}
$$

and when $t=(n+1) T$,

$$
\begin{aligned}
V\left((n+1) T^{+}\right)= & \lambda x_{1}\left((n+1) T^{+}\right)+\lambda x_{2}\left((n+1) T^{+}\right) \\
& +y_{1}\left((n+1) T^{+}\right)+y_{2}\left((n+1) T^{+}\right) \\
\leq & V((n+1) T)+\frac{a^{2}}{4 b} .
\end{aligned}
$$

It follows from the comparison theorem of impulsive differential equations (see Lemma 2.2 [21], page 23) that, for $t \in(n T,(n+1) T)$, we have

$$
\begin{array}{r}
V(t, x) \leq V\left(0^{+}\right) e^{-\mu t}+\frac{M_{1}}{\mu}\left(1-e^{-\mu t}\right)+\frac{a^{2}}{4 b} \frac{e^{-\mu(t-T)}}{1-e^{\mu T}} \\
+\frac{a^{2}}{4 b} \frac{e^{\mu T}}{e^{\mu T}-1} \longrightarrow \frac{M_{1}}{\mu}+\frac{a^{2}}{4 b} \frac{e^{\mu T}}{e^{\mu T}-1}, \\
t \rightarrow \infty .
\end{array}
$$

So $V(t, x)$ is ultimately bounded. Hence, by the definition of $V(t, x)$, there exists a constant $M>0$, such that $x_{i}(t) \leq$ $M, y_{i}(t) \leq M, i=1,2$, for $t$ large enough. The proof is complete.

Definition 6. System (3) is said to be permanent if there are constants $m, M>0$ and a finite time $T_{0}$ such that all solutions 
$x_{i}(t), y_{i}(t)$ with all initial values $x_{i}\left(0^{+}\right)>0, y_{i}\left(0^{+}\right)>0, m \leq$ $x_{i}(t) \leq M$, and $m \leq x_{i}(t) \leq M$ hold for all $t \geq T_{0}$.

\section{Extinction}

In this section, we investigate the stability of the two-pest prey eradication periodic solution as a solution of system (3). We give the condition which assures the asymptotical stability of the prey eradication periodic solution $\left(0,0, \tilde{y}_{1}(t), \tilde{y}_{2}(t)\right)$.

Theorem 7. Let $\left(x_{1}(t), x_{2}(t), y_{1}(t), y_{2}(t)\right)$ be any solution of (3); then $\left(0,0, \tilde{y}_{1}(t), \widetilde{y}_{2}(t)\right)$ is globally asymptotically stable if

$$
\begin{aligned}
& p_{4}<p_{4}^{*}, \\
& T<\min \left\{\frac{\ln \left(1 /\left(1-p_{1}\right)\right)+r_{1} y_{1}^{*} /(m+\mu)+r_{2}\left(y_{1}^{*}+y_{2}^{*}\right) / \mu}{b_{1}},\right. \\
&\left.\quad \frac{\ln \left(1 /\left(1-p_{2}\right)\right)+r_{3} y_{1}^{*} /(m+\mu)+r_{4}\left(y_{1}^{*}+y_{2}^{*}\right) / \mu}{b_{2}}\right\}
\end{aligned}
$$

hold true.

Proof. Firstly, we prove the local stability of $T$-period solution $\left(0,0, \tilde{y}_{1}(t), \tilde{y}_{2}(t)\right)$ which may be determined by considering the behavior of small-amplitude perturbations $\left(u_{1}(t), u_{2}(t), v_{1}(t), v_{2}(t)\right)$ of the solution.

Define

$$
\begin{aligned}
& x_{1}(t)=u_{1}(t), \\
& x_{2}(t)=u_{2}(t), \\
& y_{1}(t)=v_{1}(t)+\tilde{y}_{1}(t), \\
& y_{2}(t)=v_{2}(t)+\tilde{y}_{2}(t),
\end{aligned}
$$

where $u_{1}(t), u_{2}(t), v_{1}(t)$, and $v_{2}(t)$ are small perturbations; they may be written as

$$
\left(\begin{array}{l}
u_{1}(t) \\
u_{2}(t) \\
v_{1}(t) \\
v_{2}(t)
\end{array}\right)=\Phi(t)\left(\begin{array}{l}
u_{1}(0) \\
u_{2}(0) \\
v_{1}(0) \\
v_{2}(0)
\end{array}\right)
$$

where $\Phi(t)$ satisfy

$$
\frac{d \Phi(t)}{d t}=\left(\begin{array}{cccc}
b_{1}-r_{1} \tilde{y}_{1}(t)-r_{2} \tilde{y}_{2}(t) & 0 & 0 & 0 \\
0 & b_{2}-r_{3} \tilde{y}_{1}(t)-r_{4} \tilde{y}_{2}(t) & 0 & 0 \\
\lambda_{1} r_{1} \tilde{y}_{1}(t) & \lambda_{3} r_{3} \tilde{y}_{1}(t) & -(m+\mu) & 0 \\
\lambda_{2} r_{2} \tilde{y}_{2}(t) & \lambda_{4} r_{4} \tilde{y}_{2}(t) & m & -\mu
\end{array}\right) \Phi(t),
$$

with $\Phi(0)=I$, where $I$ is the identity matrix.

Hence the fundamental solution matrix is

$$
\Phi(t)=\left(\begin{array}{cccc}
\exp \left(\int_{0}^{t} H_{1} d s\right) & 0 & 0 & 0 \\
* & \exp \left(\int_{0}^{t} H_{2} d s\right) & 0 & 0 \\
* & * & \exp (-(m+\mu) t) & 0 \\
* & * & \exp (-\mu t)-\exp (-(\mu+m) t) & \exp (-\mu t)
\end{array}\right),
$$

where $H_{1}=b_{1}-r_{1} \tilde{y}_{1}(s)-r_{2} \tilde{y}_{2}(s)$ and $H_{2}=b_{2}-r_{3} \tilde{y}_{1}(s)-$ $r_{4} \tilde{y}_{2}(s)$. There is no need to calculate the exact form of $(*)$ as it is not required in the analysis that follows. The resetting impulsive conditions of (3) become

$$
\begin{aligned}
& \left(\begin{array}{c}
u_{1}\left((n+l) T^{+}\right) \\
u_{2}\left((n+l) T^{+}\right) \\
v_{1}\left((n+l) T^{+}\right) \\
v_{2}\left((n+l) T^{+}\right)
\end{array}\right) \\
& =\left(\begin{array}{cccc}
1-p_{1} & 0 & 0 & 0 \\
0 & 1-p_{2} & 0 & 0 \\
0 & 0 & 1-p_{3} & 0 \\
0 & 0 & 0 & 1-p_{4}
\end{array}\right)\left(\begin{array}{l}
u_{1}((n+l) T) \\
u_{2}((n+l) T) \\
v_{1}((n+l) T) \\
v_{2}((n+l) T)
\end{array}\right),
\end{aligned}
$$

$$
\begin{aligned}
& \left(\begin{array}{l}
u_{1}\left((n+1) T^{+}\right) \\
u_{2}\left((n+1) T^{+}\right) \\
v_{1}\left((n+1) T^{+}\right) \\
v_{2}\left((n+1) T^{+}\right)
\end{array}\right) \\
& =\left(\begin{array}{llll}
1 & 0 & 0 & 0 \\
0 & 1 & 0 & 0 \\
0 & 0 & 1 & a \\
0 & 0 & 0 & 1
\end{array}\right)\left(\begin{array}{l}
u_{1}\left((n+1) T^{+}\right) \\
u_{2}\left((n+1) T^{+}\right) \\
v_{1}\left((n+1) T^{+}\right) \\
v_{2}\left((n+1) T^{+}\right)
\end{array}\right) . \\
& \text {M } \\
& =\left(\begin{array}{cccc}
1-p_{1} & 0 & 0 & 0 \\
0 & 1-p_{2} & 0 & 0 \\
0 & 0 & 1-p_{3} & 0 \\
0 & 0 & 0 & 1-p_{4}
\end{array}\right)\left(\begin{array}{llll}
1 & 0 & 0 & 0 \\
0 & 1 & 0 & 0 \\
0 & 0 & 1 & a \\
0 & 0 & 0 & 1
\end{array}\right) \Phi(T)
\end{aligned}
$$


which are

$$
\begin{aligned}
& \lambda_{1}=\left(1-p_{1}\right) \exp \left(\int_{0}^{T}\left(b_{1}-r_{1} \tilde{y}_{1}(s)-r_{2} \tilde{y}_{2}(s)\right) d s\right), \\
& \lambda_{2}=\left(1-p_{2}\right) \exp \left(\int_{0}^{T}\left(b_{2}-r_{3} \tilde{y}_{1}(s)-r_{4} \tilde{y}_{2}(s)\right) d s\right), \\
& \lambda_{3}=\left(1-p_{3}\right)\left[e^{-(\mu+m) T}+a e^{-\mu T}\left(1-e^{-m T}\right)\right]<1, \\
& \lambda_{4}=\left(1-p_{4}\right) e^{-\mu T}<1, \\
& \lambda_{1}<1 \text { if } T \\
& <\frac{\ln \left(1 /\left(1-p_{1}\right)\right)+r_{1} y_{1}^{*} /(m+\mu)+r_{2}\left(y_{1}^{*}+y_{2}^{*}\right) / \mu}{b_{1}}, \\
& \lambda_{2}<1 \text { if } T \\
& <\frac{\ln \left(1 /\left(1-p_{2}\right)\right)+r_{3} y_{1}^{*} /(m+\mu)+r_{4}\left(y_{1}^{*}+y_{2}^{*}\right) / \mu}{b_{2}} .
\end{aligned}
$$

According to the Floquet theory (see [21]), the two-pest eradication solution $\left(0,0, \tilde{y}_{1}(t), \tilde{y}_{2}(t)\right)$ is locally asymptotically stable.

In the following, we prove the global attractivity. Choose $\varepsilon_{1}, \varepsilon_{2}>0$ such that

$$
\begin{aligned}
\eta & =\left(1-p_{3}\right) \\
& \cdot\left(\exp \left(\int_{0}^{T}\left(b_{1}-r_{1}\left(\widetilde{y}_{1}(t)-\varepsilon_{1}\right)-r_{2}\left(\widetilde{y}_{2}(t)-\varepsilon_{2}\right) d s\right)\right)\right)
\end{aligned}
$$

$<1$.

Noting that $\dot{y}_{1}(t) \geq-(m+\mu) y_{1}(t), \dot{y}_{2}(t) \geq m y_{1}(t)-\mu y_{2}(t)$, consider the following impulsive differential equation:

$$
\begin{aligned}
& \dot{z}_{1}(t)=-(m+\mu) z_{1}(t), \\
& \dot{z}_{2}(t)=m z_{1}(t)-\mu z_{2}(t),
\end{aligned}
$$$$
t \neq(n+l) T, t \neq(n+1) T,
$$

$$
\begin{aligned}
& \Delta z_{1}(t)=-p_{3} z_{1}(t), \\
& \Delta z_{2}(t)=-p_{4} z_{2}(t),
\end{aligned}
$$

$$
\begin{aligned}
& \Delta z_{1}(t)=z_{2}(t)\left(a-b z_{2}(t)\right), \\
& \Delta z_{2}(t)=0,
\end{aligned}
$$

$$
t=(n+l) T,
$$

It follows from impulsive differential equation (30) that $y_{1}(t) \geq z_{1}(t), y_{2}(t) \geq z_{2}(t)$ and $z_{1}(t) \rightarrow \tilde{y}_{1}(t), z_{2}(t) \rightarrow \tilde{y}_{2}(t)$ as $t \rightarrow \infty$. This is

$$
\begin{aligned}
& y_{1}(t) \geq z_{1}(t) \geq \tilde{y}_{1}(t)-\varepsilon_{1}, \\
& y_{2}(t) \geq z_{2}(t) \geq \tilde{y}_{2}(t)-\varepsilon_{2},
\end{aligned}
$$

for all $t$ large enough. For convenience, we may assume that (31) holds. From (3) and (31) we have

$$
\begin{aligned}
& \begin{array}{l}
\dot{x}_{1}(t) \leq x_{1}(t)\left(b_{1}-r_{1}\left(\tilde{y}_{1}(t)-\varepsilon_{1}\right)-r_{2}\left(\tilde{y}_{2}(t)-\varepsilon_{2}\right)\right), \\
t
\end{array} \\
& x_{1}\left(t^{+}\right)=(n+l) T, \\
&
\end{aligned}
$$

holding for all $t$ large enough. Integrate (32) on $((n+$ $l) T,(n+1) T]$, which yields $x_{1}((n+l) T) \leq x_{1}\left(n T^{+}\right)(1-$ $\left.p_{1}\right) \exp \left(\int_{n T}^{(n+l) T}\left(b_{1}-r_{1}\left(\tilde{y}_{1}(s)-\varepsilon_{1}\right)-r_{2}\left(\tilde{y}_{2}(s)-\varepsilon_{2}\right)\right) d s\right)$. Thus $x_{1}((n+l) T) \leq x_{1}\left(l T^{+}\right) \eta^{n}$ and $x_{1}((n+l) T) \rightarrow 0$ as $t \rightarrow \infty$. Therefore $x_{1}(t) \rightarrow 0$ as $t \rightarrow \infty$, since $0<x_{1}(t)<$ $x_{1}((n+l) T)\left(1-p_{1}\right) \exp \left(b_{1} T\right)$ for $(n+l) T \leq t \leq(n+1) T$. By the same method we can prove $x_{2}(t) \rightarrow 0$, as $t \rightarrow \infty$, so we omit it.

Next, we will prove that $y_{1}(t) \rightarrow \tilde{y}_{1}(t), y_{2}(t) \rightarrow \tilde{y}_{2}(t)$, as $t \rightarrow \infty$. If $\lim _{t \rightarrow \infty} x_{1}(t)=0$ and $\lim _{t \rightarrow \infty} x_{2}(t)=0$, for $0<\varepsilon \leq(m+\mu) /\left(\lambda_{1} r_{1}+\lambda_{3} r_{3}\right)$, there exists $t_{0}>0$ such that $0<x_{1}(t)<\varepsilon_{1}$ and $0<x_{2}(t)<\varepsilon$, for all $t>t_{0}$; then we have

$$
\begin{aligned}
& -(m+\mu) y_{1}(t) \leq \dot{y}_{1}(t) \\
& \quad \leq-\left[(m+\mu)-\lambda_{1} r_{1} \varepsilon-\lambda_{3} r_{3} \varepsilon\right] y_{1}(t), \\
& m y_{1}(t)-\mu y_{2}(t) \leq \dot{y}_{2}(t) \\
& \quad \leq m y_{1}(t)-\left(\mu-\lambda_{2} r_{2} \varepsilon-\lambda_{4} r_{4} \varepsilon\right) y_{2}(t) .
\end{aligned}
$$

From the left hand inequality of (33), it follows from impulsive differential equation (5) that $y_{1}(t) \rightarrow \widetilde{y}_{1}(t)$ and $y_{2}(t) \rightarrow \tilde{y}_{2}(t)$ as $t \rightarrow \infty$. For the right hand inequality, we consider the following impulsive differential equation:

$$
\begin{array}{rlrl}
\dot{z}_{3}(t) & =-\left[(m+\mu)-\lambda_{1} r_{1} \varepsilon-\lambda_{3} r_{3} \varepsilon\right] z_{3}(t), & \\
\dot{z}_{4}(t) & =m z_{3}(t)-\left(\mu-\lambda_{2} r_{2} \varepsilon-\lambda_{4} r_{4} \varepsilon\right) z_{4}(t), \\
t \neq(n+l) T, & t \neq(n+1) T, \\
\Delta z_{3}(t) & =-p_{3} z_{3}(t), & t \\
\Delta z_{4}(t) & =-p_{4} z_{4}(t), & \\
\Delta z_{3}(t) & \left.=z_{4}(t)\left(a-b z_{4}(t)\right), l\right) T, \\
\Delta z_{4}(t) & =0, & t &
\end{array}
$$

System (34) has a globally asymptotically stable positive periodic solution $\left(\widetilde{z}_{3}(t), \widetilde{z}_{4}(t)\right)$, where 


$$
\begin{aligned}
& \tilde{z}_{3}(t)= \begin{cases}z_{3}^{*} e^{-\left(m+\mu-\lambda_{1} r_{1} \varepsilon-\lambda_{3} r_{3} \varepsilon\right)(t-n T)}, & t \in(n T,(n+l) T], \\
z_{3}^{*} e^{-\left(m+\mu-\lambda_{1} r_{1} \varepsilon-\lambda_{3} r_{3} \varepsilon\right) l T} e^{-\left(m+\mu-\lambda_{1} r_{1} \varepsilon-\lambda_{3} r_{3} \varepsilon\right)(t-(n+l) T)}, & t \in((n+l) T,(n+1) T],\end{cases} \\
& \widetilde{z}_{4}(t) \\
& = \begin{cases}e^{-\left(\mu-\lambda_{2} r_{2} \varepsilon-\lambda_{4} r_{4} \varepsilon\right)(t-n T)}\left[z_{4}^{*}+z_{3}^{*}\left(1-e^{-m(t-n T)}\right)\right], & t \in(n T,(n+l) T], \\
e^{-\left(\mu-\lambda_{2} r_{2} \varepsilon-\lambda_{4} r_{4} \varepsilon\right)(t-(n+l) T)}\left\{e^{-\left(\mu-\lambda_{2} r_{2} \varepsilon-\lambda_{4} r_{4} \varepsilon\right) l T}\left[z_{4}^{*}+z_{3}^{*}\left(1-e^{-m l T}\right)\right]+z_{3}^{*} e^{-\left(m+\mu-\lambda_{1} r_{1} \varepsilon-\lambda_{3} r_{3} \varepsilon\right) l T}\left(1-e^{-m(t-(n+l) T)}\right)\right\}, & t \in((n+l) T,(n+1) T],\end{cases}
\end{aligned}
$$

where

$$
z_{4}^{*}=\frac{\left(B_{1}+a C_{1}-1-A_{1} B_{1}+A_{1}\right)}{b C_{1}}, \quad p_{4}<p_{4}^{\star},
$$

$$
z_{3}^{*}=\frac{\left(B_{1}+a C_{1}-1-A_{1} B_{1}+A_{1}\right)\left(1-A_{1}\right)}{b C_{1}^{2}}
$$

$$
\text { and } A_{1}=e^{-\left(\mu-\lambda_{2} r_{2} \varepsilon-\lambda_{4} r_{4} \varepsilon\right) T}\left(1-p_{4}\right)>0, B_{1}=
$$
$\left(1-p_{3}\right) e^{-\left(m+\mu-\lambda_{1} r_{1} \varepsilon-\lambda_{3} r_{3} \varepsilon\right) T}>0$, and $C_{1}=(1-$ $\left.p_{4}\right) e^{-\left(\mu-\lambda_{2} r_{2} \varepsilon-\lambda_{4} r_{4} \varepsilon\right) T}\left(1-e^{-m l T}\right)+\left(1-p_{3}\right) e^{-\left(m+\mu-\lambda_{1} r_{1} \varepsilon-\lambda_{3} r_{3} \varepsilon\right) T}(1-$ $\left.e^{(1-l) m T}\right)>0$.

$$
p_{4}<p_{4}^{\star}
$$

Set

$$
p_{4}^{\star}=\frac{e^{-\theta_{1} T} e^{\theta_{2} T}\left(1-p_{3}\right)+\left(1-\mu_{1}\right)\left(1-e^{-m T-m l T}\right) a-e^{\theta_{2} T}+1+a\left(1-e^{-m T}\right)-\left(1-p_{3}\right) e^{-\theta_{1} T}}{1+a\left(1-e^{-m T}\right)-\left(1-p_{3}\right) e^{-\theta_{1} T}},
$$

where $\theta_{1}=m+\mu-\lambda_{1} r_{1} \varepsilon-\lambda_{3} r_{3} \varepsilon$ and $\theta_{2}=\mu-\lambda_{2} r_{2} \varepsilon-\lambda_{4} r_{4} \varepsilon$.

Therefore, there exists $\varepsilon_{1}>0$ such that

$$
\widetilde{z}_{1}(t)-\varepsilon_{1}<y_{1}(t)<\widetilde{z}_{3}(t)+\varepsilon_{1}
$$

for $t$ large enough; let $\varepsilon \rightarrow 0$; then we get $\widetilde{z}_{3}(t) \rightarrow \widetilde{y}_{1}(t)$, $\tilde{z}_{1}(t) \rightarrow \tilde{y}_{1}(t)$. Hence $y_{1}(t) \rightarrow \tilde{y}_{1}(t)$ as $t \rightarrow \infty$. Similarly, we can prove $y_{2}(t) \rightarrow \tilde{y}_{2}(t)$ as $t \rightarrow \infty$, so we omit it. This proof is complete.

\section{Permanence}

In this section, we will investigate the permanence of system (3). In biological terms, the permanence implies that preys and predators will coexist, none of them facing extinction or growing indefinitely.

Theorem 8. System (3) is permanent if $p_{4}<p_{4}^{*}, b_{1}>\alpha b_{2}$, and $b_{2}>\beta b_{1}$,

$$
\begin{aligned}
& -\frac{r_{1} y_{1}^{*} e^{-\left(m+\mu-r_{3} \lambda_{3} b_{2}\right) l T}\left(e^{-\left(m+\mu-r_{3} \lambda_{3} b_{2}\right) T}-1\right)}{-\left(m+\mu-r_{3} \lambda_{3} b_{2}\right)}-\frac{r_{2} y_{1}^{*} e^{-\left(m+\mu-r_{3} \lambda_{3} b_{2}\right) l T}\left(e^{-\left(\mu-r_{4} \lambda_{4} b_{2}+m\right) T}-1\right)}{-\left(\mu-r_{4} \lambda_{4} b_{2}\right)-m}-\ln \frac{1}{1-p_{1}} \\
& -\frac{r_{2}\left(e^{-\left(\mu-r_{4} \lambda_{4} b_{2}\right) T}-1\right)\left\{e^{-\left(\mu-r_{4} \lambda_{4} b_{2}\right) l T}\left[y_{1}^{*}\left(1-e^{-m l T}\right)+y_{2}^{*}\right]+y_{1}^{*} e^{-\left(m+\mu-r_{3} \lambda_{3} b_{2}\right) l T}\right\}}{-\left(\mu-r_{4} \lambda_{4} b_{2}\right)}+\left(b_{1}-\alpha b_{2}\right) T>0, \\
& -\frac{r_{3} y_{1}^{*} e^{-\left(m+\mu-r_{3} \lambda_{3} b_{2}\right) l T}\left(e^{-\left(m+\mu-r_{3} \lambda_{3} b_{2}\right) T}-1\right)}{-\left(m+\mu-r_{3} \lambda_{3} b_{2}\right)}-\frac{r_{4} y_{1}^{*} e^{-\left(m+\mu-r_{3} \lambda_{3} b_{2}\right) l T}\left(e^{-\left(\mu-r_{4} \lambda_{4} b_{2}+m\right) T}-1\right)}{-\left(\mu-r_{4} \lambda_{4} b_{2}\right)-m}-\ln \frac{1}{1-p_{2}} \\
& -\left(\mu-r_{4} \lambda_{4} b_{2}\right) \\
& -\frac{r_{4}\left(e^{-\left(\mu-r_{4} \lambda_{4} b_{2}\right) T}-1\right)\left\{e^{-\left(\mu-r_{4} \lambda_{4} b_{2}\right) l T}\left[y_{1}^{*}\left(1-e^{-m l T}\right)+y_{2}^{*}\right]+y_{1}^{*} e^{-\left(m+\mu-r_{3} \lambda_{3} b_{2}\right) l T}\right\}}{-\left(b_{2}-\beta b_{1}\right) T>0 .}
\end{aligned}
$$


Proof. Let us suppose a solution $x(t)=$ $\left(x_{1}(t), x_{2}(t), y_{1}(t), y_{2}(t)\right)$ of (3) with initial value $x(t)>0$. From Lemma 5, we have proved that there exists a constant $M>0$ such that $x_{i}(t) \leq M, y_{i}(t) \leq M(i=1,2)$, and $M>\max \left\{b_{1} /\left(r_{1}+r_{2}\right), b_{2} /\left(r_{3}+r_{4}\right)\right\}$ for $t>0$. Note that

$$
\begin{aligned}
\dot{x}_{1}(t) & \leq x_{1}(t)\left(b_{1}-x_{1}(t)\right), \quad t \neq(n+l) T, \\
\Delta x_{1}(t) & =-p_{1} x_{1}(t), \quad t=(n+l) T, \\
x_{1}\left(0^{+}\right) & \geq 0 .
\end{aligned}
$$

Consider the following comparison equation:

$$
\begin{aligned}
\dot{\omega}_{1}(t) & =\omega_{1}(t)\left(b_{1}-\omega_{1}(t)\right), \quad t \neq(n+l) T, \\
\Delta \omega_{1}(t) & =-p_{1} \omega_{1}(t), \quad t=(n+l) T, \\
\omega_{1}\left(0^{+}\right) & =\omega_{1}\left(0^{+}\right) \geq 0 .
\end{aligned}
$$

We have $x_{1}(t) \leq \omega_{1}(t)$ and $\omega_{1}(t) \rightarrow b_{1}$ as $t \rightarrow \infty$; thus there exists $\varepsilon_{3}^{\prime}$ such that $x_{1}(t)<b_{1}+\varepsilon_{3}^{\prime}$ for $t$ large enough. Without loss of generality, we may assume $x_{1}(t)<b_{1}+\varepsilon_{3}^{\prime}$ for $t>0$. Similarly, we may set $x_{2}(t)<b_{2}+\varepsilon_{4}^{\prime}$. From (31), we know $y_{1}(t)>\widetilde{z}_{1}(t)-\varepsilon, y_{2}(t)>\widetilde{z}_{2}(t)-\varepsilon$ for all $t$ large enough $(\varepsilon>0$ is enough small), so that $y_{1}(t) \geq y_{1}^{*} e^{-(m+\mu) T}-\varepsilon \triangleq m_{3}, y_{2}(t) \geq$ $e^{-\mu T}\left(y_{2}^{*}+y_{1}^{*}\left(1-e^{-m l T}\right)\right)+y_{1}^{*} e^{-(\mu+m l) T}\left(1-e^{-m(1-l) T}\right)-\varepsilon \triangleq m_{4}$ for $t$ large enough. Thus we only need to find $\bar{m}_{1}>0, \bar{m}_{2}>0$ such that $x_{1}(t) \geq \bar{m}_{1}, x_{2}(t) \geq \bar{m}_{2}$ for $t$ large enough. We will do it in the following two steps.

Step 1. Denote

$$
\begin{gathered}
0<m_{1}<\min \left\{\frac{(m+\mu)-r_{3} \lambda_{3}\left(b_{2}+\varepsilon_{4}^{\prime}\right)}{r_{1} \lambda_{1}},\right. \\
\left.\frac{\mu-m-r_{4} \lambda_{4}\left(b_{2}+\varepsilon_{4}^{\prime}\right)}{r_{2} \lambda_{2}}\right\},
\end{gathered}
$$

$$
\begin{aligned}
& \dot{z}_{6}(t)=-\omega_{1} z_{6}(t), \\
& \dot{z}_{7}(t)=m z_{7}(t)-\omega_{2} z_{7}(t),
\end{aligned}
$$

$\Delta z_{6}(t)=-p_{3} z_{6}(t)$,$$
\Delta z_{7}(t)=-p_{4} z_{7}(t) \text {, }
$$$$
\Delta z_{6}(t)=z_{7}(t)\left(a-b z_{7}(t)\right),
$$$$
\Delta z_{7}(t)=0 \text {, }
$$$$
\widetilde{z}_{6}(t)= \begin{cases}z_{6}^{*} e^{-\omega_{1}(t-n T)}, & t \in(n T,(n+l) T], \\ z_{6}^{*} e^{-\omega_{1} l T} e^{-\omega_{1}(t-(n+l) T)}, & t \in((n+l) T,(n+1) T],\end{cases}
$$$$
\widetilde{z}_{7}(t)= \begin{cases}e^{-\omega_{2}(t-n T)}\left[z_{7}^{*}+z_{6}^{*}\left(1-e^{-m(t-n T)}\right)\right], & t \in(n T,(n+l) T], \\ e^{-\omega_{2}(t-(n+l) T)}\left\{e^{-\omega_{2} l T}\left[z_{7}^{*}+z_{6}^{*}\left(1-e^{-m l T}\right)\right]+z_{6}^{*} e^{-\omega_{1} l T}\left(1-e^{-m(t-(n+l) T)}\right)\right\}, & t \in((n+l) T,(n+1) T],\end{cases}
$$

$$
\begin{gathered}
0<m_{2}<\min \left\{\frac{(m+\mu)-r_{1} \lambda_{1}\left(b_{1}+\varepsilon_{4}^{\prime}\right)}{r_{3} \lambda_{3}},\right. \\
\left.\frac{\mu-m-r_{2} \lambda_{2}\left(b_{1}+\varepsilon_{4}^{\prime}\right)}{r_{4} \lambda_{4}}\right\}
\end{gathered}
$$

and $r_{1} \lambda_{1} m_{1}+r_{3} \lambda_{3} m_{2}>m+\mu$. We will prove that there exist $t_{1}, t_{2} \in(0, \infty)$, such that $x_{1}\left(t_{1}\right) \geq m_{1}$ and $x_{2}\left(t_{2}\right) \geq m_{2}$. Otherwise there will be three cases:

(i) There exists $t_{2}>0$, such that $x_{2}\left(t_{2}\right) \geq m_{2}$ and $x_{1}(t)<$ $m_{1}$ for all $t>0$.

(ii) There exists $t_{1}>0$, such that $x_{1}\left(t_{1}\right) \geq m_{1}$ and $x_{2}(t)<$ $m_{2}$ for all $t>0$.

(iii) Consider $x_{1}(t)<m_{1}, x_{2}(t)<m_{2}$, for all $t>0$.

Firstly, consider case (i). Let $\varepsilon_{1}>0$ be small enough, so that

$$
\begin{aligned}
\sigma & =\left(b_{1}-m_{1}-\alpha\left(b_{2}+\varepsilon_{4}^{\prime}\right)-r_{1} \varepsilon_{1}-r_{2} \varepsilon_{1}\right) T \\
& -\frac{r_{1} z_{6}^{*} e^{-\omega_{1} l T}\left(e^{-\omega_{1} T}-1\right)}{\left(-\omega_{1}\right)}-\ln \frac{1}{1-p_{1}}>0 \\
& -\frac{\left(e^{-\omega_{2} T}-1\right)\left\{e^{-\omega_{2} l T}\left[z_{6}^{*}\left(1-e^{-m l T}\right)+z_{7}^{*}\right]+z_{6}^{*} e^{-\omega_{1} l T}\right\}}{\left(-\omega_{2}\right)} \\
& -\frac{z_{6}^{*} e^{-\omega_{1} l T}\left(e^{-\left(\varrho_{2}+m\right) T}-1\right)}{\left(-\omega_{2}-m\right)},
\end{aligned}
$$

where

$$
\begin{aligned}
& \omega_{1}=(m+\mu)-r_{1} \lambda_{1} m_{1}-r_{3} \lambda_{3}\left(b_{2}+\varepsilon_{4}^{\prime}\right), \\
& \omega_{2}=\mu-r_{2} \lambda_{2} m_{1}-r_{4} \lambda_{4}\left(b_{2}+\varepsilon_{4}^{\prime}\right) .
\end{aligned}
$$

According to the above assumption, it is easy to get that $\dot{y}_{1}(t) \leq-y_{1}(t) \omega_{1}$ and $\dot{y}_{2}(t) \leq m y_{1}(t)-\omega_{2} y_{2}(t)$. Then we consider the following impulsive differential equation:

$$
t \neq(n+l) T, t \neq(n+1) T,
$$

$$
t=(n+1) T
$$


where

$$
z_{6}^{*}=\frac{\left(B_{2}+a C_{2}-1-A_{2} B_{2}+A_{2}\right)\left(1-A_{2}\right)}{b C_{2}^{2}},
$$$$
z_{7}^{*}=\frac{\left(B_{2}+a C_{2}-1-A_{2} B_{2}+A_{2}\right)}{b C_{2}}, \quad p_{4}<p_{4}^{*}
$$

and $A_{2}=e^{-\omega_{1} T}\left(1-p_{4}\right)>0, B_{2}=\left(1-p_{3}\right) e^{-\omega_{1} T}>0, C_{2}=$ $p_{4}<p_{4}^{*}$,

$$
p_{4}^{*}=\frac{e^{-\omega_{1} T} e^{\omega_{2} T}\left(1-p_{3}\right)+\left(1-\mu_{1}\right)\left(1-e^{-m T-m l T}\right) a-e^{\varrho_{2} T}+1+a\left(1-e^{-m T}\right)-\left(1-p_{3}\right) e^{-\Phi_{1} T}}{1+a\left(1-e^{-m T}\right)-\left(1-p_{3}\right) e^{-\omega_{1} T}} .
$$

Therefore, there exists $T_{1}>0$ such that

$$
\begin{aligned}
& y_{1}(t) \leq z_{6}(t) \leq \widetilde{z}_{6}(t)+\varepsilon_{1}, \\
& y_{2}(t) \leq z_{7}(t) \leq \widetilde{z}_{7}(t)+\varepsilon_{1} .
\end{aligned}
$$

$$
\Delta x_{1}(t)=-p_{1} x_{1}(t), \quad t=(n+l) T,
$$

Thus we can easily get

$$
\dot{x}_{1}(t) \geq x_{1}(t)\left(b_{1}-m_{1}-\alpha\left(b_{2}+\varepsilon_{4}^{\prime}\right)-r_{1}\left(\widetilde{z}_{6}(t)+\varepsilon_{1}\right)\right.
$$

for $t>T_{1}$. Set $(N+l-1) T \geq T_{1}, N \in Z_{+}$. Integrating (49) on $((n+l) T,(n+1) T], n \geq N$, then we obtain

$$
\begin{aligned}
& x_{1}((n+l+1) T) \\
& \quad \geq x_{1}\left((n+l) T^{+}\right)\left(1-p_{1}\right) \exp \left(\int_{(n+l) T}^{(n+l+1) T}\left(b_{1}-m_{1}-\alpha\left(b_{2}+\varepsilon_{4}^{\prime}\right)-r_{1}\left(\widetilde{z}_{6}(t)+\varepsilon_{1}\right)-r_{2}\left(\widetilde{z}_{7}(t)+\varepsilon_{1}\right)\right)\right) d t \\
& \quad=x_{1}((n+l) T) e^{\sigma} ;
\end{aligned}
$$

consequently $x_{1}((N+n+l) T) \geq x_{1}((N+l) T) e^{n \sigma} \rightarrow \infty$ as $n \rightarrow \infty$, which contradicts to the boundedness of $x_{1}(t)$.

Similarly, cases (ii) and (iii) can be analyzed as in case (i). Here we omit it. From the above three cases, we conclude that there exist $t_{1}>0, t_{2}>0$ such that $x_{1}\left(t_{1}\right) \geq m_{1}, x_{2}\left(t_{2}\right) \geq m_{2}$.

Step 2. If $x_{1}(t) \geq m_{1}$ for all $t \geq t_{1}$, then our aim is obtained. Otherwise, $x_{1}(t)<m_{1}$ for any $t \geq t_{1}$. Let $\widetilde{t}=\inf _{t>t_{1}}\left\{x_{1}(t)<\right.$ $\left.m_{1}\right\}$; there are two possible cases for $\widetilde{t}$.

Case 1. Consider $\tilde{t}=\left(n_{1}+l\right) T, n_{1} \in Z_{+}$. Then $x_{1}(t) \geq m_{1}$ for $t \in\left[t_{1}, \widetilde{t}\right]$ and $\left(1-p_{1}\right) m_{1} \leq$ $x_{1}\left(\widetilde{t}^{+}\right)=\left(1-p_{1}\right) x_{1}(\widetilde{t})<m_{1}$. Choose $n_{2}, n_{3} \in Z_{+}$ such that

$$
\begin{aligned}
& \left(n_{2}-1\right) T>\max \left\{\frac{\ln \left(\varepsilon_{1} / M\right)}{-\varrho_{1}}, \frac{\ln \left(\left(\varepsilon_{1} / M\right)\right)}{-\Phi_{2}}\right\}, \\
& \left(1-p_{1}\right)^{n_{2}} e^{\sigma^{n_{3}}} e^{\left(n_{2} \eta_{3} T\right)}>1 .
\end{aligned}
$$

Set $T^{\prime}=n_{2} T+n_{3} T$; we claim that there must exist $t_{2} \in(\widetilde{t}, \tilde{t}+$ $\left.T^{\prime}\right)$ such that $x_{1}\left(t_{2}\right)>m_{1}$. In view of (45), with $z_{6}\left(n_{1} T^{+}\right)=$ $y_{1}\left(n_{1} T^{+}\right), z_{7}\left(n_{1} T^{+}\right)=y_{2}\left(n_{1} T^{+}\right)$, we have

$$
\begin{aligned}
& z_{6}(t)= \begin{cases}z_{6}\left(n_{1} T^{+}\right) e^{-\left((m+\mu)+r_{1} \lambda_{1} m_{1}+r_{3} \lambda_{3}\left(b_{2}+\varepsilon_{4}^{\prime}\right)\right)(t-n T)}+\widetilde{z}_{6}(t), & t \in(n T,(n+l) T], \\
z_{6}\left(\left(n_{1}+l\right) T^{+}\right) e^{-\left((m+\mu)+r_{1} \lambda_{1} m_{1}+r_{3} \lambda_{3}\left(b_{2}+\varepsilon_{4}^{\prime}\right)\right)(t-(n+l) T)}+\widetilde{z}_{6}(t), & t \in((n+l) T,(n+1) T],\end{cases} \\
& z_{7}(t) \\
& = \begin{cases}e^{-\left(\mu-r_{2} \lambda_{2} m_{1}-r_{4} \lambda_{4}\left(b_{2}+\varepsilon_{4}^{\prime}\right)\right)(t-n T)}\left[z_{7}\left(n_{1} T^{+}\right)+z_{6}\left(n_{1} T^{+}\right)\left(1-e^{-m(t-n T)}\right)\right]+\widetilde{z}_{7}(t), & t \in(n T,(n+l) T], \\
e^{-\left(\mu-r_{2} \lambda_{2} m_{1}-r_{4} \lambda_{4}\left(b_{2}+\varepsilon_{4}^{\prime}\right)\right)(t-(n+l) T)}\left[z_{7}\left(\left(n_{1}+l\right) T^{+}\right)+z_{6}\left(\left(n_{1}+l\right) T^{+}\right)\left(1-e^{-m(t-(n+l) T)}\right)\right]+\widetilde{z}_{7}(t), & t \in((n+l) T,(n+1) T] .\end{cases}
\end{aligned}
$$


And $n_{1}+1 \leq n \leq n_{1}+n_{2}+n_{3}$. Then

$$
\begin{aligned}
& \left|z_{6}(t)-\widetilde{z}_{6}(t)\right|<M \\
& \quad \cdot \exp \left(-\left((m+\mu)+r_{1} \lambda_{1} m_{1}+r_{3} \lambda_{3}\left(b_{2}+\varepsilon_{4}^{\prime}\right)\right)\right. \\
& \cdot(t-n T))<\varepsilon_{1}, \\
& \left|z_{7}(t)-\widetilde{z}_{7}(t)\right|<M \\
& \quad \cdot \exp \left(-\left(\mu-r_{2} \lambda_{2} m_{1}-r_{4} \lambda_{4}\left(b_{2}+\varepsilon_{4}^{\prime}\right)\right)\right)<\varepsilon_{1} ;
\end{aligned}
$$

thus $y_{1}(t) \leq z_{6}(t)<\widetilde{z}_{6}(t)+\varepsilon_{1}$ and $y_{2}(t) \leq z_{7}(t)<\widetilde{z}_{7}(t)+\varepsilon_{1}$ for $n_{1} T+\left(n_{2}-1\right) T \leq t \leq \widetilde{t}+T^{\prime}$, which implies that (49) holds for $\tilde{t}+n_{2} T \leq t \leq \tilde{t}+T^{\prime}$. So as in step 1 , we get

$$
x_{1}\left(\tilde{t}+T^{\prime}\right) \geq x_{1}\left(\tilde{t}+n_{2} T\right) e^{\sigma^{n_{3}}} .
$$

From system (3), we get

$$
\begin{array}{r}
\dot{x}_{1}(t) \geq x_{1}(t)\left(b_{1}-m_{1}-\alpha\left(b_{2}+\varepsilon_{4}^{\prime}\right)-r_{1} \mathrm{M}-r_{2} M\right), \\
t \neq(n+l) T,
\end{array}
$$

$$
\Delta x_{1}(t)=-p_{1} x_{1}(t), \quad t=(n+l) T .
$$

Integrating (55) on $\left[\widetilde{t}, \widetilde{t}+n_{2} T\right]$, we have

$$
x_{1}\left(\tilde{t}+n_{2} T\right) \geq m_{1}\left(1-p_{1}\right)^{n_{2}} \exp \left(n_{2} \eta_{3} T\right)
$$

Therefore, we deduce that

$$
x_{1}\left(\tilde{t}+T^{\prime}\right) \geq m_{1}\left(1-p_{1}\right)^{n_{2}} e^{\sigma^{n_{3}}} e^{\left(n_{2} \eta_{3} T\right)}>m_{1},
$$

which is a contradiction.

Let $\widehat{t}=\inf _{t>\widetilde{t}}\left\{x_{1}(t) \geq m_{1}\right\}$; then for $t \in(\widetilde{t}, \hat{t}), x_{1}(t)<m_{1}$ and $x_{1}(\hat{t})=m_{1}$. For $t \in(\widetilde{t}, \hat{t})$, set $t \in(\widetilde{t}+(r-1) T, \tilde{t}+r T]$, $r \in Z_{+}, r \leq n_{2}+n_{3}$; from (55), we have

$$
\begin{aligned}
& x_{1}(t) \geq x_{1}\left(\tilde{t}^{+}\right)\left(1-p_{1}\right)^{r-1} \exp \left((r-1) \eta_{3} T\right) \\
& \cdot \exp \left(\eta_{3}(t-(\widetilde{t}+(r-1) T))\right) \geq m_{1}\left(1-p_{1}\right)^{r}
\end{aligned}
$$

$$
\begin{aligned}
& \cdot \exp \left(r \eta_{3} T\right) \geq m_{1}\left(1-p_{1}\right)^{n_{2}+n_{3}} \\
& \cdot \exp \left(\left(n_{2}+n_{3}\right) \eta_{3} T\right) .
\end{aligned}
$$

Let $\widehat{m}_{1}=m_{1}\left(1-p_{1}\right)^{n_{2}+n_{3}} \exp \left(\left(n_{2}+n_{3}\right) \eta_{3} T\right)$, so we have $x_{1}(t) \geq \widehat{m}_{1}$ for $\left.t \in \widetilde{t}, \widehat{t}\right)$. For $t>\widehat{t}$, the same argument can be continued since $x_{1}(t) \geq m_{1}$.

Case 2. Consider $\tilde{t} \neq(n+l) T, n \in Z_{+}$. Then $x_{1}(t) \geq m_{1}$ for $t \in\left[t_{1}, \widetilde{t}\right)$ and $x_{1}(\widetilde{t})=m_{1}$. Let $\tilde{t} \in\left(\left(n_{1}^{\prime}+l\right) T,\left(n_{1}^{\prime}+1\right) T\right)$, $n_{1}^{\prime} \in Z_{+}$. There are two possible cases for $\left.t \in \widetilde{t},\left(n_{1}^{\prime}+l\right) T\right)$.

If $x_{1}(t)<m_{1}$ for all $t \in\left(\widetilde{t},\left(n_{1}^{\prime}+1\right) T\right)$, there must be $t_{2}^{\prime} \epsilon$ $\left[\left(n_{1}^{\prime}+1\right) T,\left(n_{1}^{\prime}+1\right) T+T^{\prime}\right]$ such that $x_{1}\left(t_{2}^{\prime}\right) \geq m_{1}$. Here, we omit it.

Let $\check{t}=\inf _{t>\widetilde{t}}\left\{x_{1}(t) \geq m_{1}\right\}$; then for $t \in(\widetilde{t}, \check{t}), x_{1}(t)<m_{1}$ and $x_{1}(\check{t})=m_{1}$. For $t \in(\widetilde{t}, \check{t})$, let $t \in\left(\left(n_{1}^{\prime}+l\right) T+\left(r^{\prime}-1\right) T,\left(n_{1}^{\prime}+\right.\right.$ l) $\left.T+r^{\prime} T\right], r^{\prime} \in Z_{+}, r^{\prime} \leq n_{2}+n_{3}$; from (55), we have

$$
\begin{aligned}
x_{1}(t) & \geq m_{1}\left(1-p_{1}\right)^{r^{\prime}} \exp \left(\left(r^{\prime}+1\right) \eta_{3} T\right) \\
& \geq m_{1}\left(1-p_{1}\right)^{n_{2}+n_{3}} \exp \left(\left(n_{2}+n_{3}+1\right) \eta_{3} T\right) .
\end{aligned}
$$

Let $\bar{m}_{1}=m_{1}\left(1-p_{1}\right)^{n_{2}+n_{3}} \exp \left(\left(n_{2}+n_{3}+1\right) \eta_{3} T\right)$, so we have $x_{1}(t) \geq \bar{m}_{1}$ for $t \in(t, \check{t})$. For $t>\check{t}$, the same argument can be continued since $x_{1}(\check{t}) \geq m_{1}$.

If there exists $t \in\left(\widetilde{t},\left(n_{1}^{\prime}+l\right) T\right)$ such that $x_{1}(t) \geq m_{1}$, let $\bar{t}=\inf _{t>\tilde{t}}\left\{x_{1}(t) \geq m_{1}\right\}$; then $x_{1}(t)<m_{1}$ for $t \in(\tilde{t}, \bar{t})$ and $x_{1}(\check{t})=m_{1}$. For $t \in(\tilde{t}, \bar{t})$, (55) holds; integrating (55) on $(\tilde{t}, \bar{t})$, we have

$$
x_{1}(t) \geq x_{1}(\tilde{t}) \exp \left(\eta_{3}(t-\widetilde{t})\right) \geq m_{1} \exp \left(\eta_{3} T\right)>\bar{m}_{1} .
$$

Since $x_{1}(\bar{t}) \geq m_{1}$ for $t>\bar{t}$, the same argument can be continued. Hence, we have $x_{1}(t) \geq \bar{m}_{1}$ for all $t>t_{1}$. Similarly, we can prove $x_{2}(t) \geq \bar{m}_{2}$ for all $t \geq t_{2}$. The proof is complete.

From the proof of Theorems 7 and 8, we can derive the following results.

Corollary 9. Let $\left(x_{1}(t), x_{2}(t), y_{1}(t), y_{2}(t)\right)$ be any solution of (3); then $y_{i}, i=1,2$, are permanent; $x_{1}(t) \rightarrow 0$ as $t \rightarrow \infty$ provided, $b_{1}>\alpha b_{2}, b_{2}>\beta b_{1}$,

$$
\begin{aligned}
& T<\frac{\ln \left(1 /\left(1-p_{2}\right)\right)+r_{3} y_{1}^{*} /(m+\mu)+r_{4}\left(y_{1}^{*}+y_{2}^{*}\right) / \mu}{b_{2}}, \\
& -\frac{r_{1} y_{1}^{*} e^{-\left(m+\mu-r_{3} \lambda_{3} b_{2}\right) l T}\left(e^{-\left(m+\mu-r_{3} \lambda_{3} b_{2}\right) T}-1\right)}{-\left(m+\mu-r_{3} \lambda_{3} b_{2}\right)}-\frac{r_{2} y_{1}^{*} e^{-\left(m+\mu-r_{3} \lambda_{3} b_{2}\right) l T}\left(e^{-\left(\left(\mu-r_{4} \lambda_{4} b_{2}\right)+m\right) T}-1\right)}{-\left(\mu-r_{4} \lambda_{4} b_{2}\right)-m}-\ln \frac{1}{1-p_{1}} \\
& -\frac{r_{2}\left(e^{-\left(\mu-r_{4} \lambda_{4} b_{2}\right) T}-1\right)\left\{e^{-\left(\mu-r_{4} \lambda_{4} b_{2}\right) l T}\left[y_{1}^{*}\left(1-e^{-m l T}\right)+y_{2}^{*}\right]+y_{1}^{*} e^{-\left(m+\mu-r_{3} \lambda_{3} b_{2}\right) l T}\right\}}{-\left(\mu-r_{4} \lambda_{4} b_{2}\right)}+\left(b_{1}-\alpha b_{2}\right) T>0 .
\end{aligned}
$$




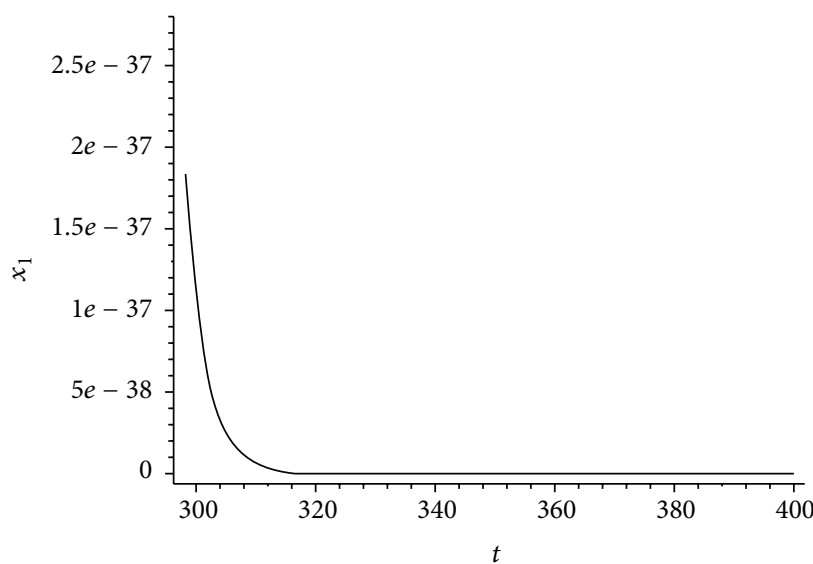

(a)

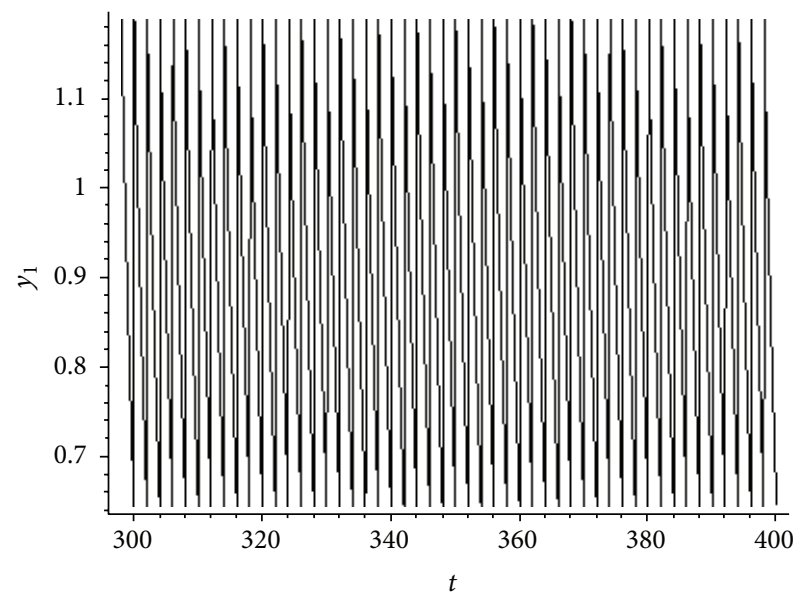

(c)

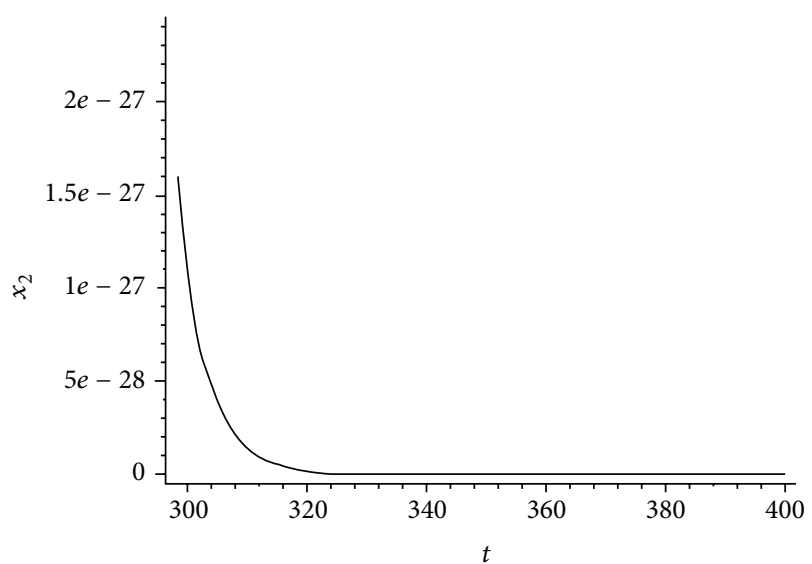

(b)

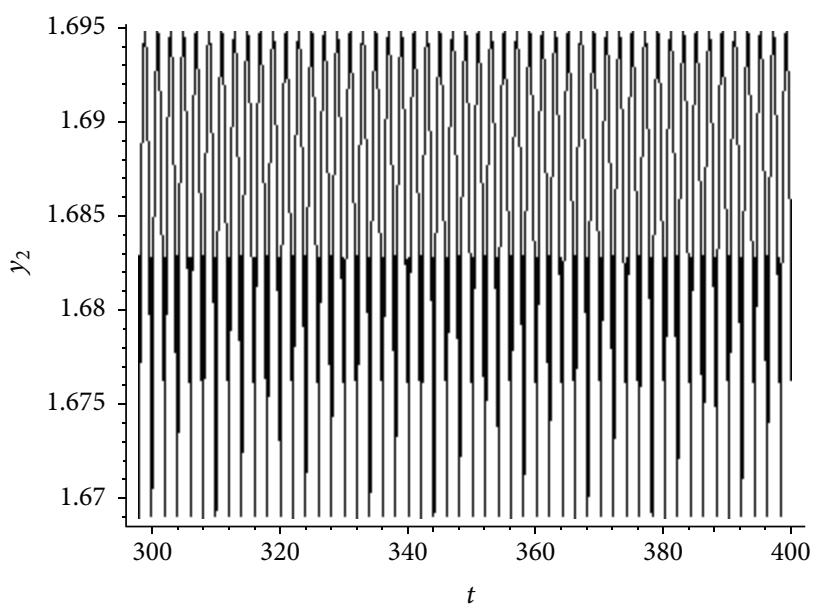

(d)

FIGURE 1: Globally asymptotically stable prey eradication solution of system (3). (a, b) Time series of prey populations $x_{1}(t), x_{2}(t)$ and $(\mathrm{c}, \mathrm{d})$ time series of predator populations $y_{1}(t), y_{2}(t)$ with initial values $x_{1}(0)=0.5, x_{2}(0)=0.5, y_{1}(0)=0.5$, and $y_{2}(0)=0.5$.

Corollary 10. Let $\left(x_{1}(t), x_{2}(t), y_{1}(t), y_{2}(t)\right)$ be any solution of (3); then $x_{1}$ and $y_{i}, i=1,2$, are permanent; $x_{2}(t) \rightarrow 0$ as $t \rightarrow \infty$ provided, $b_{1}>\alpha b_{2}, b_{2}>\beta b_{1}$,

$$
\begin{aligned}
T & <\frac{\ln \left(1 /\left(1-p_{1}\right)\right)+r_{1} y_{1}^{*} /(m+\mu)+r_{2}\left(y_{1}^{*}+y_{2}^{*}\right) / \mu}{b_{1}}, \\
- & \frac{r_{3} y_{1}^{*} e^{-\left(m+\mu-r_{3} \lambda_{3} b_{2}\right) l T}\left(e^{-\left(m+\mu-r_{3} \lambda_{3} b_{2}\right) T}-1\right)}{-\left(m+\mu-r_{3} \lambda_{3} b_{2}\right)}-\frac{r_{4} y_{1}^{*} e^{-\left(m+\mu-r_{3} \lambda_{3} b_{2}\right) l T}\left(e^{-\left(\left(\mu-r_{4} \lambda_{4} b_{2}\right)+m\right) T}-1\right)}{-\left(\mu-r_{4} \lambda_{4} b_{2}\right)-m}-\ln \frac{1}{1-p_{2}} \\
& -\frac{r_{4}\left(e^{-\left(\mu-r_{4} \lambda_{4} b_{2}\right) T}-1\right)\left\{e^{-\left(\mu-r_{4} \lambda_{4} b_{2}\right) l T}\left[y_{1}^{*}\left(1-e^{-m l T}\right)+y_{2}^{*}\right]+y_{1}^{*} e^{-\left(m+\mu-r_{3} \lambda_{3} b_{2}\right) l T}\right\}}{-\left(\mu-r_{4} \lambda_{4} b_{2}\right)}+\left(b_{2}-\beta b_{1}\right) T>0 .
\end{aligned}
$$

\section{Numerical Analysis}

In this section, we carry out numerical simulations of system (3) to illustrate and support our analytical results.
(1) Consider the following choice of parametric values: $\alpha=0.3, \beta=0.2, b_{1}=1, b_{2}=1, r_{1}=0.2, r_{2}=0.5, r_{3}=0.3$, $r_{4}=0.4, \lambda_{1}=0.1, \lambda_{2}=0.2, \lambda_{3}=0.3, \lambda_{4}=0.4, m=0.2$, 


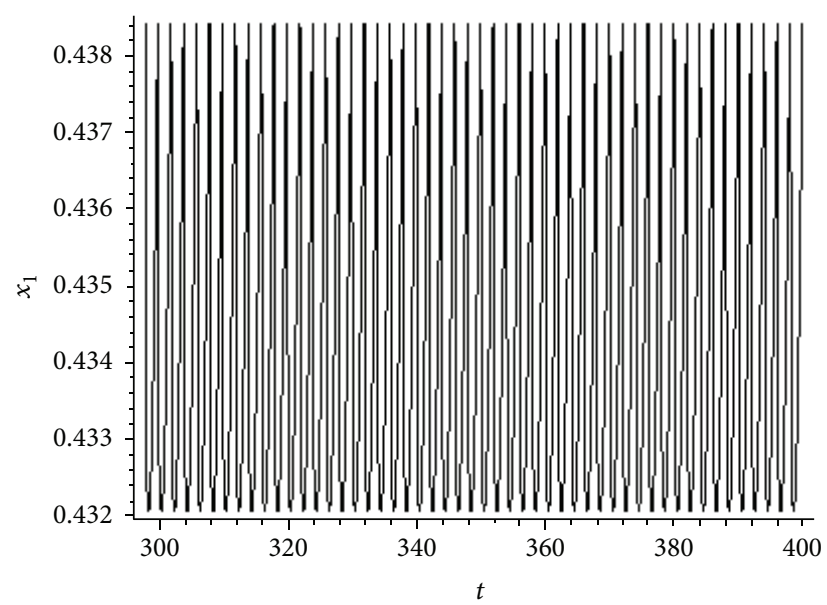

(a)

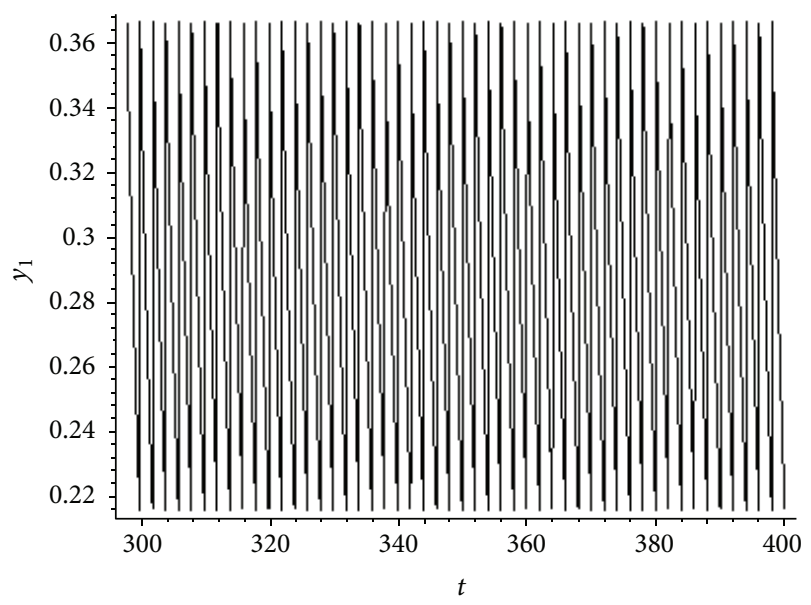

(c)

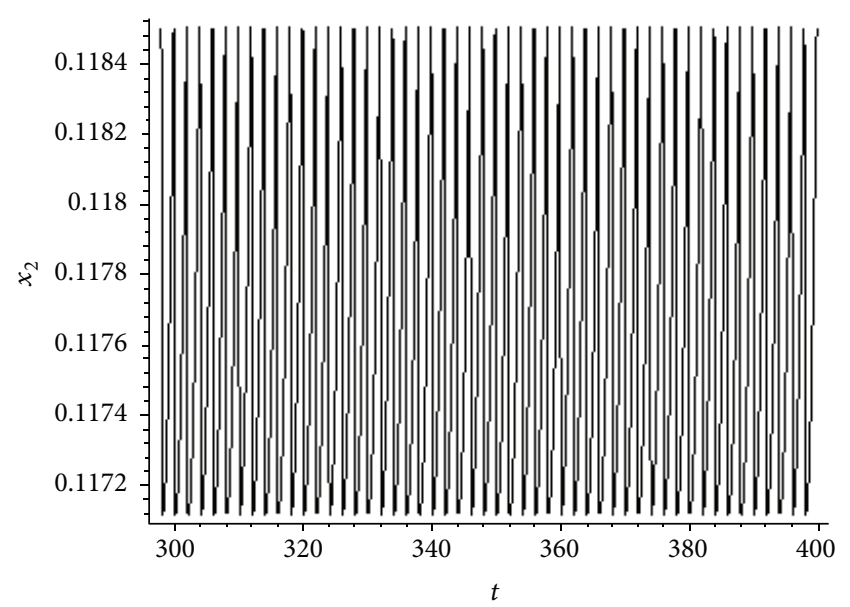

(b)

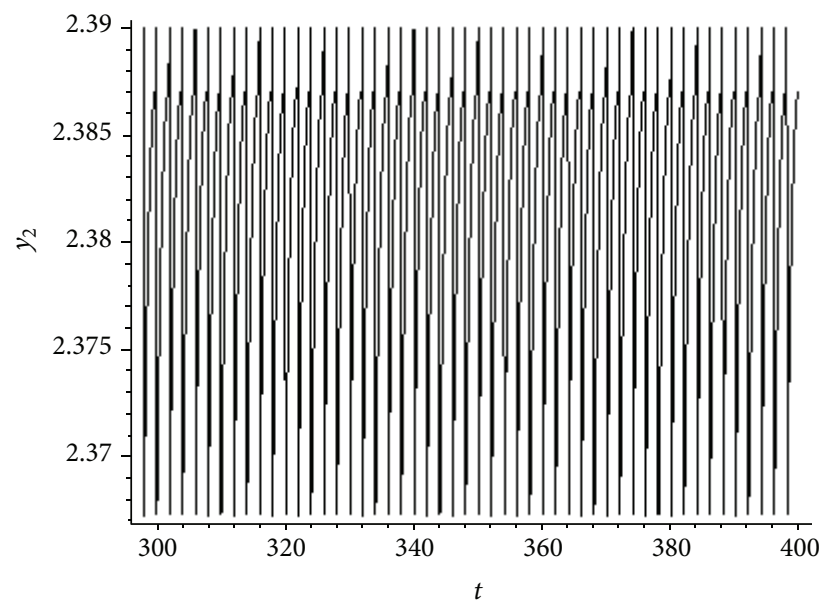

(d)

FIGURE 2: The permanence of system (3). (a, b) Time series of prey populations $x_{1}(t), x_{2}(t)$ and (c, d) time series of predator populations $y_{1}(t), y_{2}(t)$ with initial values $x_{1}(0)=0.5, x_{2}(0)=0.5, y_{1}(0)=0.5$, and $y_{2}(0)=0.5$.

$\mu=0.1, d=0.4, p_{1}=0.01, p_{2}=0.01, p_{3}=0.01, p_{4}=0.01$, $a=2, b=1, l=0.6$, and $T=2$.

Correspondingly, $\min \left\{\left(\ln \left(1 /\left(1-p_{1}\right)\right)+r_{1} y_{1}^{*} /(m+\mu)+\right.\right.$ $\left.r_{2}\left(y_{1}^{*}+y_{2}^{*}\right) / \mu\right) / b_{1},\left(\ln \left(1 /\left(1-p_{2}\right)\right)+r_{3} y_{1}^{*} /(m+\mu)+r_{4}\left(y_{1}^{*}+\right.\right.$ $\left.\left.\left.y_{2}^{*}\right) / \mu\right) / b_{2}\right\} \approx 5.07>T=2$ and $p_{4}=0.01<p_{4}^{*} \approx 0.4$. According to Theorem 7 , we know that the prey eradication periodic solution of system (3) is globally asymptotically stable for this case (see Figure 1).

(2) Consider the following set of parameters: $\alpha=0.3, \beta=$ $0.2, b_{1}=1.5, b_{2}=1.5, r_{1}=0.2, r_{2}=0.5, r_{3}=0.3, r_{4}=0.4$, $\lambda_{1}=0.1, \lambda_{2}=0.2, \lambda_{3}=0.3, \lambda_{4}=0.4, m=0.2, \mu=0.1$, $d=0.4, p_{1}=0.01, p_{2}=0.01, p_{3}=0.01, p_{4}=0.01, a=2.45$, $b=1, l=0.6$, and $T=2$.

Since conditions of Theorem 8 hold for this choice, system (3) is permanent (see Figure 2).

\section{Discussion}

In this paper, we study an impulsively controlled threespecies prey-predator model with stage structure and birth pulse for predator. Using the comparison theorems, we have shown that there exists a globally asymptotically stable twopest eradication periodic solution when the impulsive period $T<\min \left\{\left(\ln \left(1 /\left(1-p_{1}\right)\right)+r_{1} y_{1}^{*} /(m+\mu)+r_{2}\left(y_{1}^{*}+\right.\right.\right.$ $\left.\left.\left.y_{2}^{*}\right) / \mu\right) / b_{1},\left(\ln \left(1 /\left(1-p_{2}\right)\right)+r_{3} y_{1}^{*} /(m+\mu)\right) / b_{2}+r_{4}\left(y_{1}^{*}+y_{2}^{*}\right) / \mu b_{2}\right\}$. In biological terms, the pest population is eradicated totally and the predator population will tend to a stable level. However, the prey population is eradicated totally, which is not most desired for us from the biological point and saving resources; we hope to control the species under the economic threshold level or drive the target prey population to extinction and let the nontarget pest (or harmless) be permanent. Therefore, we give the sufficient conditions for the permanence of the system. What is more, the sufficient conditions for the extinction of one of the two prey species and permanence of the remaining other preys and predators are given in Corollaries 9 and 10 .

Compared to earlier modeling studies on the preypredator concerning chemical control for pest control at different fixed time, our model considers the predator given birth in regular pules at $t=(n+1) T, n=1,2, \ldots$. To avoid the fact that pesticides impact on the newborn predator, 
pesticide applications and birth pulse in predator occur at different time in this paper. All these results show that the dynamical behaviors of system (3) become more complex under different time periodically impulsive effect. Our results suggest a new approach in pest control. We are more interested in the following: how different patterns of insecticide applications affect the results, for instance, applying pesticide after predator is born and applying pesticide when predator is born. We also can study the results of two cases and find the better control way. These works will be done in our next consideration.

\section{Conflict of Interests}

The authors declare that there is no conflict of interests regarding the publication of this paper.

\section{Acknowledgments}

This work is supported by Key Laboratory of Biologic Resources Protection and Utilization of Hubei Province (PKLHB1524), the innovation projects for undergraduates of Hubei province (201310517014), the soft science research project of Hubei province (2012GDA01309), and key discipline of Hubei province, Forestry.

\section{References}

[1] T. K. Kar and U. K. Pahari, "Modelling and analysis of a preypredator system with stage-structure and harvesting," Nonlinear Analysis: Real World Applications, vol. 8, no. 2, pp. 601-609, 2007.

[2] H. Wang and W. Wang, "The dynamical complexity of a Ivlev-type prey-predator system with impulsive effect," Chaos, Solitons \& Fractals, vol. 38, no. 4, pp. 1168-1176, 2008.

[3] L. Ling and W. M. Wang, "Dynamics of a Ivlev-type predatorprey system with constant rate harvesting," Chaos, Solitons \& Fractals, vol. 41, no. 4, pp. 2139-2153, 2009.

[4] W. J. Qin, Z. J. Liu, and Y. P. Chen, "Permanence and global stability of positive periodic solutions of a discrete competitive system," Discrete Dynamics in Nature and Society, vol. 2009, Article ID 830537, 13 pages, 2009.

[5] J. J. Jiao and Y. L. Liu, "Stability and bifurcation analysis of a delayed Leslie-Gower predator-prey system with nonmonotonic functional response," Abstract and Applied Analysis, vol. 2013, Article ID 152459, 19 pages, 2013.

[6] A. J. Terry, "Impulsive adult culling of a tropical pest with a stage-structured life cycle," Nonlinear Analysis: Real World Applications, vol. 11, no. 2, pp. 645-664, 2010.

[7] W. Wang and L. Chen, "A predator-prey system with stagestructure for predator," Computers \& Mathematics with Applications, vol. 33, no. 8, pp. 83-91, 1997.

[8] J. D. Parrish and S. B. Saila, "Interspecific competition, predation and species diversity," Journal of Theoretical Biology, vol. 27, no. 2, pp. 207-220, 1970.

[9] L. P. Pedigo, Entomology and Pest Management, Prentice-Hall, Englewood Cliffs, NJ, USA, 1998.

[10] Y. Takeuchi and N. Adachi, "Existence and bifurcation of stable equilibrium in two-prey, one-predator communities," Bulletin of Mathematical Biology, vol. 45, no. 6, pp. 877-900, 1983.
[11] K. Y. Liu, X. Z. Meng, and L. S. Chen, "A new stage structured predator-prey Gomportz model with time delay and impulsive perturbations on the prey," Applied Mathematics and Computation, vol. 196, no. 2, pp. 705-719, 2008.

[12] W. G. Aiello and H. I. Freedman, "A time-delay model of singlespecies growth with stage structure," Mathematical Biosciences, vol. 101, no. 2, pp. 139-153, 1990.

[13] C.-Y. Huang, Y.-J. Li, and H.-F. Huo, "The dynamics of a stage-structured predator-prey system with impulsive effect and Holling mass defence," Applied Mathematical Modelling, vol. 36, no. 1, pp. 87-96, 2012.

[14] J. H. Liang, S. Y. Tang, and R. A. Cheke, "An integrated pest management model with delayed responses to pesticide applications and its threshold dynamics," Nonlinear Analysis. Real World Applications, vol. 13, no. 5, pp. 2352-2374, 2012.

[15] S. Tang and R. A. Cheke, "Models for integrated pest control and their biological implications," Mathematical Biosciences, vol. 215, no. 1, pp. 115-125, 2008.

[16] Z. Y. Xiang and X. Y. Song, "Extinction and permanence of a two-prey two-predator system with impulsive on the predator," Chaos, Solitons \& Fractals, vol. 29, no. 5, pp. 1121-1136, 2006.

[17] M. G. Roberts and R. R. Kao, "The dynamics of an infectious disease in a population with birth pulses," Mathematical Biosciences, vol. 149, no. 1, pp. 23-36, 1998.

[18] S. Tang and L. Chen, "Density-dependent birth rate, birth pulses and their population dynamic consequences," Journal of Mathematical Biology, vol. 44, no. 2, pp. 185-199, 2002.

[19] V. Lakshmikantham, D. D. Bainov, and P. S. Simeonov, Theory of Impulsive Differential Equations, World Scientific, Singapore, 1989.

[20] R. M. May, Theoretical Ecology, Principles and Application, Blackwell, Oxford, UK, 2nd edition, 1981.

[21] V. Lakshmikantham, Theory of Impulsive Differential Equations, World Scientific, Singapore, 1989. 


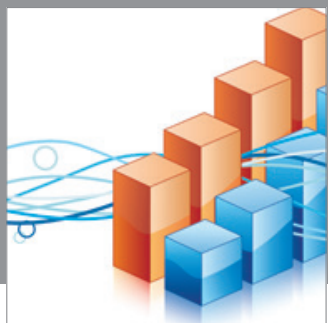

Advances in

Operations Research

mansans

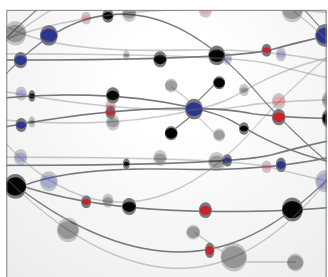

The Scientific World Journal
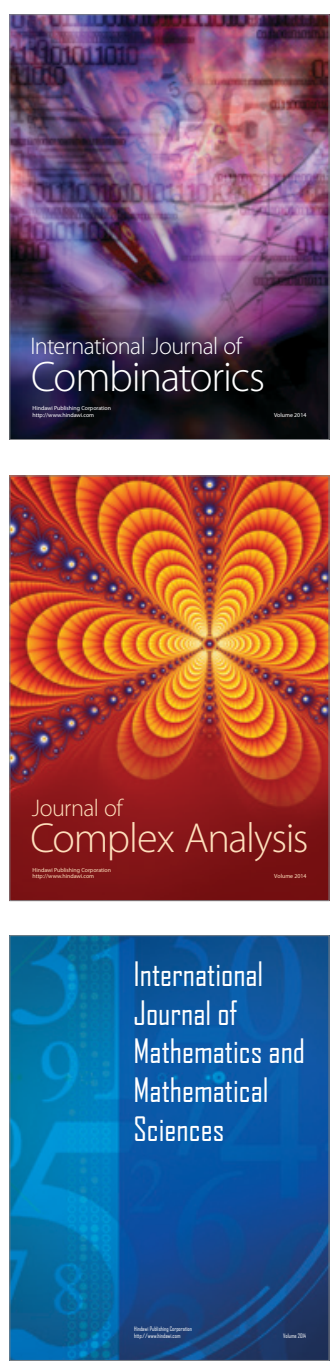
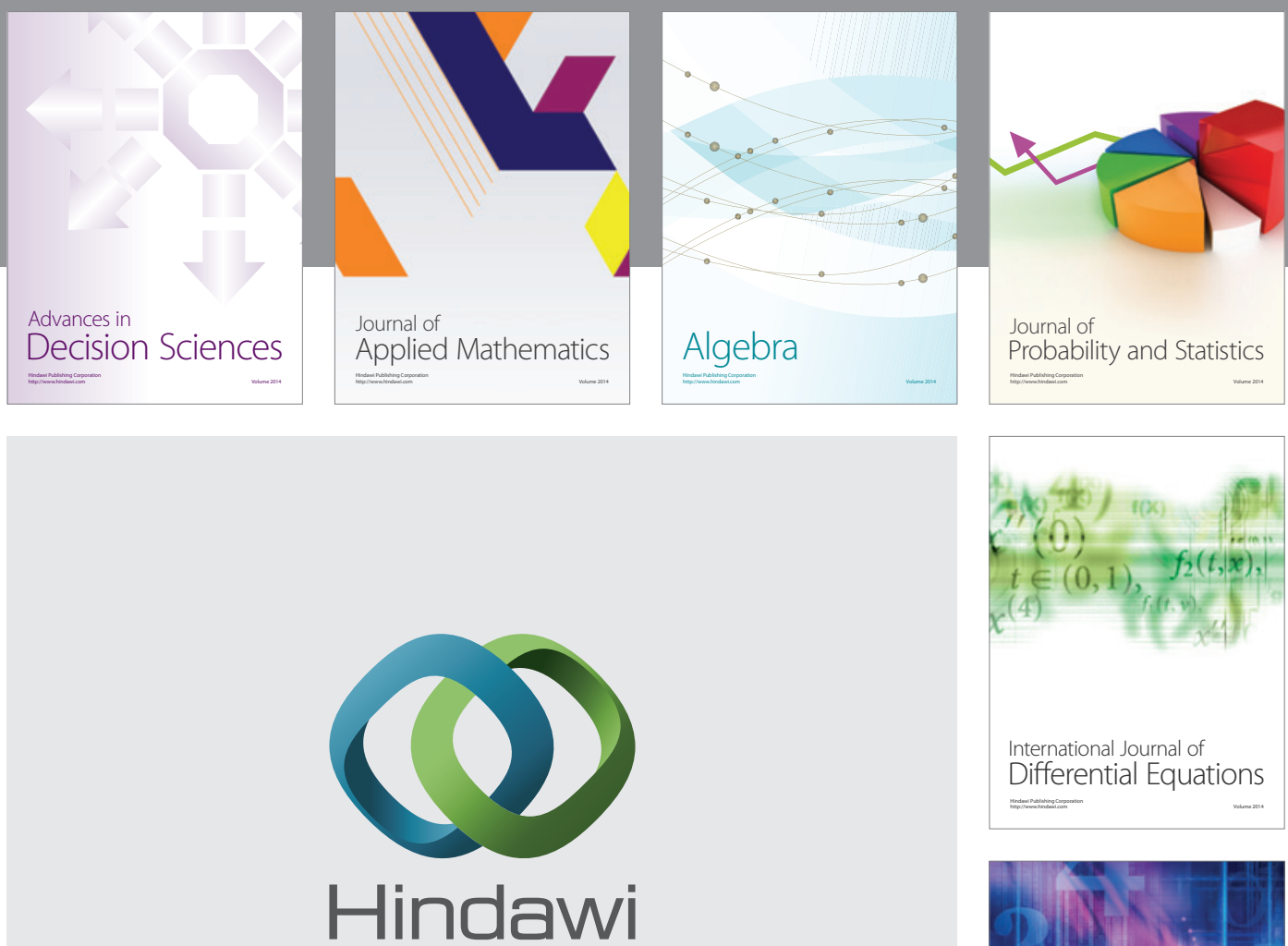

Submit your manuscripts at http://www.hindawi.com
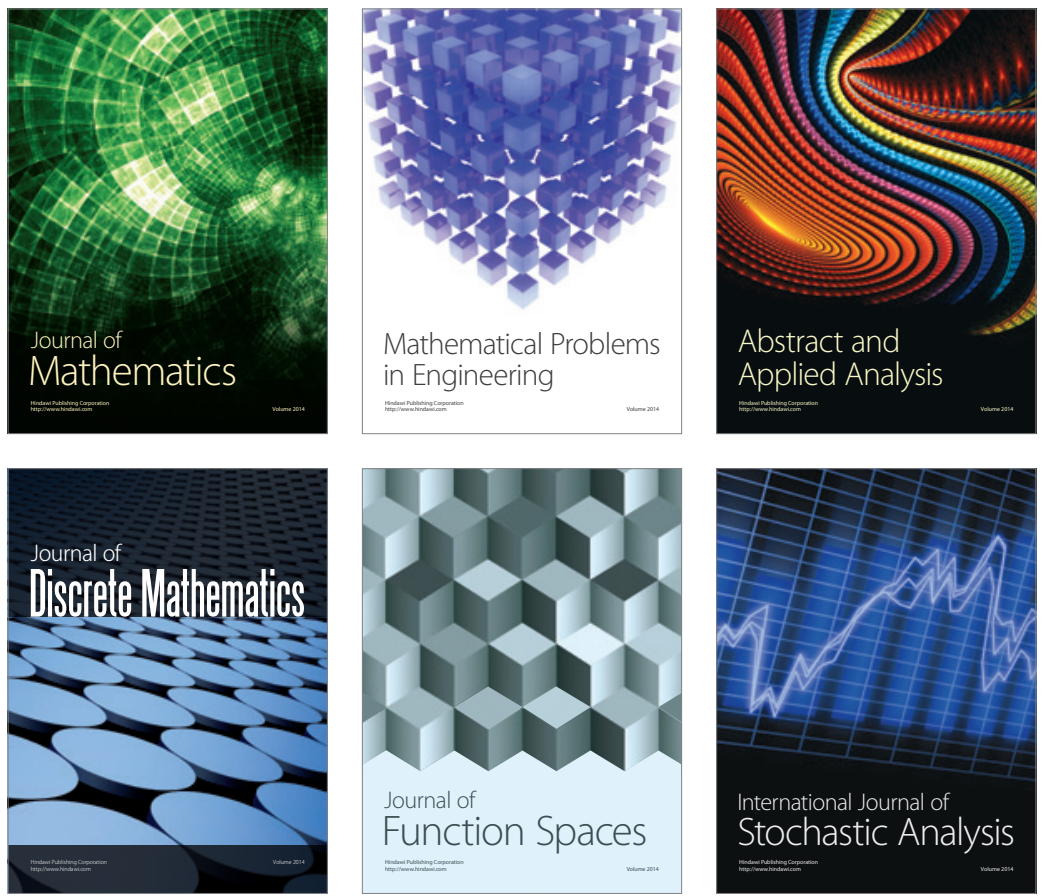

Journal of

Function Spaces

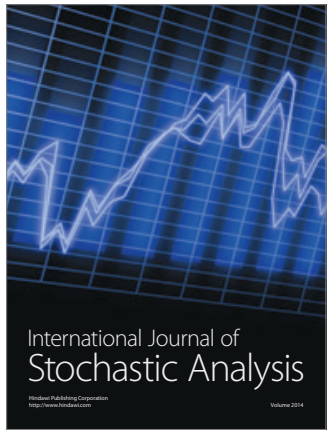

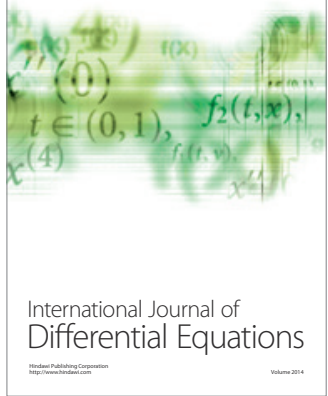
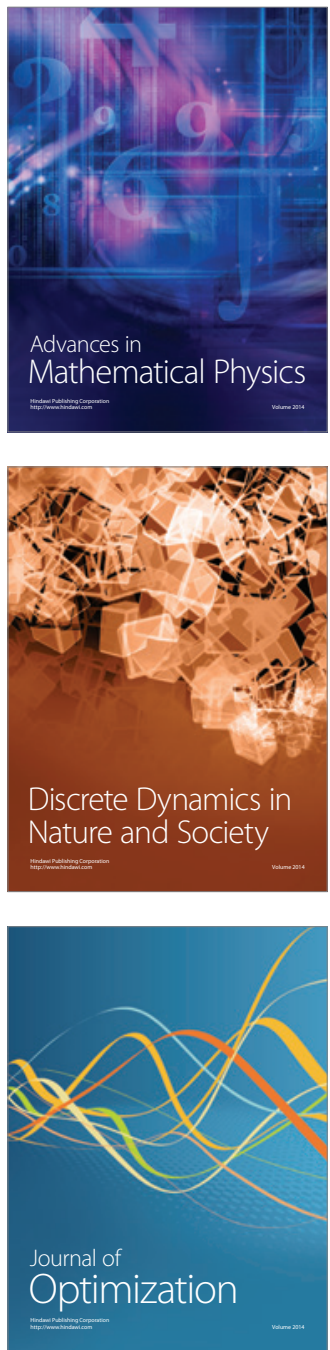